\title{
ASYMPTOTIC BEHAVIOUR OF THE DENSITY IN A PARABOLIC SPDE*
}

\author{
By A. Kohatsu-Higa ${ }^{1}$, D. Márquez-Carreras ${ }^{2}$ \\ and M. Sanz-Solé 2 \\ Universitat Pompeu Fabra, Universitat de Barcelona \\ and Universitat de Barcelona
}

Consider the density of the solution $X(t, x)$ of a stochastic heat equation with small noise at a fixed $t \in[0, T], x \in[0,1]$. In the paper we study the asymptotics of this density as the noise is vanishing. A kind of Taylor expansion in powers of the noise parameter is obtained. The coefficients and the residue of the expansion are explicitly calculated.

In order to obtain this result some type of exponential estimates of tail probabilities of the difference between the approximating process and the limit one is proved. Also a suitable local integration by parts formula is developped.

Key words and phrases: Malliavin Calculus, parabolic SPDE, large deviations, Taylor expansion of a density, exponential estimates of the tail probabilities, stochastic integration by parts formula.

Journal of Economic Literature Classification System:C15

\footnotetext{
${ }^{1}$ This work was partially supported by the DGICYT grant PB95-0978. It was completed while visiting the Departament of Mathematics at University of Tokyo.

${ }^{2}$ This work has been partially supported by the grant PB 960088 from the Subdirección General de Formación y Promoción de Conocimiento and the grant ERBFMRX CT960075A of the European Union.

${ }^{*}$ This article has also appeared as a Mathematics Preprint Series 257 of the Universitat de Barcelona.
} 


\section{Introduction}

Consider the stochastic heat equation

$$
\frac{\partial X}{\partial t}(t, x)=\frac{\partial^{2} X}{\partial x^{2}}(t, x)+\sigma(X(t, x)) \dot{W}_{t, x}+b(X(t, x)),
$$

$(t, x) \in[0, T] \times[0,1]$, with initial condition $X(0, x)=X_{0}(x)$ and either Neumann or Dirichlet boundary conditions. The process $\left\{\dot{W}_{t, x},(t, x) \in\right.$ $[0, T] \times[0,1]\}$ is a space-time white noise and $\sigma, b: \mathbb{R} \longrightarrow \mathbb{R}$ are smooth functions. The purpose of this paper is to study the Taylor expansion in $\varepsilon$ of the density $p_{t, x}^{\varepsilon}(y)$ of the probability law of the solution of (1.1) when the coefficient $\sigma$ is perturbed by a factor $\varepsilon>0$. More precisely, let $\left\{X^{\varepsilon}(t, x),(t, x) \in[0, T] \times[0,1]\right\}$ be the mild solution of (1.1), that is,

$$
\begin{aligned}
X^{\varepsilon}(t, x) & =\int_{0}^{1} G_{t}(x, y) X_{0}(y) d y \\
& +\int_{0}^{t} \int_{0}^{1} G_{t-s}(x, y)\left\{\varepsilon \sigma\left(X^{\varepsilon}(s, y)\right) W(d s, d y)+b\left(X^{\varepsilon}(s, y)\right) d s d y\right\},
\end{aligned}
$$

where $G_{t}(x, y)$ is the fundamental solution of the heat equation on $[0, T] \times$ $[0,1]$ with the above-mentioned boundary conditions. Assuming smoothness of the coefficients and strong ellipticity (see assumptions (H1), (H2) in Section 2) [Bally and Pardoux (1998)] have proved the existence and smoothness of the density of the solution of $(1.2)$ for fixed $(t, x) \in(0, T] \times(0,1], \varepsilon \in$ $(0,1]$.

Let $\mathcal{H}$ denote the reproducing kernel Hilbert space of the Wiener sheet $W=\left\{W_{t, x},(t, x) \in[0, T] \times[0,1]\right\}$. For any $h \in \mathcal{H}$, let $\left\{\Psi_{X_{0}}^{h}(t, x),(t, x) \in\right.$ $[0, T] \times[0,1]\}$ be the solution of the deterministic evolution equation

$$
\begin{aligned}
\Psi_{X_{0}}^{h}(t, x) & =\int_{0}^{1} G_{t}(x, y) X_{0}(y) d y \\
& +\int_{0}^{t} \int_{0}^{1} G_{t-s}(x, y)\left\{\sigma\left(\Psi_{X_{0}}^{h}(s, y)\right) \dot{h}(s, y)+b\left(\Psi_{X_{0}}^{h}(s, y)\right)\right\} d s d y
\end{aligned}
$$

In the previous work [Márquez-Carreras and Sanz-Solé (1998b)] we have proved a Taylor expansion of $p_{t, x}^{\varepsilon}(y)$, when $y=\Psi_{X_{0}}^{0}(t, x)$. Our purpose here is to extend this result to any $y \in \mathbb{R}$.

This problem has been extensively studied for diffusions and is related to the behaviour at small time of the density. A good list of references is given in 
[Watanabe (1987)] and [Takanobu and Watanabe (1993)]. In this situation, if $y$ is the initial value of a $d$-dimensional diffusion, say $y=x$, the zero order term of the expansion is, up to constants, $\varepsilon^{-d}$. For $y \neq x$, it is of the order $\exp \left\{-C / \varepsilon^{2}\right\} \varepsilon^{-d}$. We obtain the same behaviour for our family $\left\{X^{\varepsilon}(t, x), \varepsilon \in(0,1]\right\}$.

The method used to approach the problem is inspired in [Léandre (1988)], [Watanabe (1987)] and [Takanobu and Watanabe (1993)]. A basic assumption, formulated as (H3) in Section 3, ensures that, for any $y \in \mathbb{R}$, there is a finite number of elements $h_{1}, \cdots, h_{n_{0}}$ in $\mathcal{H}$ such that $\Psi_{X_{0}}^{h_{i}}(t, x)=y, i=$ $1, \cdots, n_{0}$, and $\left\|h_{i}\right\|_{\mathcal{H}}^{2}$ is minimum. This allows to split the problem into two parts by means of a localization around these minima. This method is developed in Section 3. Large deviations estimates for the family $\left\{X^{\varepsilon}(t, x), \varepsilon \in\right.$ $(0,1]\}$, proved in [Sowers (1992)] and [Chenal and Millet (1997)], allow us to deal with the trajectories for which $X^{\varepsilon}(t, x)$ and $\Psi_{X_{0}}^{h_{i}}(t, x), i=1, \cdots, n_{0}$, are distant. When they are close, we use Girsanov's theorem and a normalization by $\varepsilon$ to reduce the problem to the study of a Wiener functional which is the product of two random variables, one of exponential type and $f\left(\hat{X}^{\varepsilon, h}(t, x)\right)$, where $f$ is smooth and

$$
\hat{X}^{\varepsilon, h}(t, x):=\frac{X^{\varepsilon}(t, x)\left(w+\frac{h}{\varepsilon}\right)-\Psi_{X_{0}}^{h}(t, x)}{\varepsilon} .
$$

In Section 2 we prove the regularity in $\varepsilon$ which is needed in Section 3 in order to obtain the Taylor expansion of $f\left(\hat{X}^{\varepsilon, h}(t, x)\right)$. We combine this with the expansion of the exponential random variable. By means of the integration by parts formula, in the framework of Malliavin Calculus, well-suited to our problem (see Propositions $3.8,3.9$ ) we remove the derivatives of $f$ of order $k \geq 1$. The Taylor expansion is obtained by taking a sequence of smooth functions $f$ converging to the Dirac $\delta$-function $\delta_{\{0\}}$.

In the case of diffusions, the analogue of the derivatives $\frac{d^{j}}{d \varepsilon^{3}} \hat{X}^{\varepsilon, h}(t, x)$ satisfy linear equations which have an explicit solution. Furthermore one can apply the martingale representation theorem when necessary. These two ingredients are essential tools for the proof. In our case, these linear equations do not have an explicit solution and the stochastic integrals in the equations do not define martingales. These are the main causes of most the difficulties that we have encountered.

To solve these problems we compute in Section 3, exponential estimates of the tail probabilities of the processes involved. Later, in order to obtain an explicit expansion of the density we carry out a careful study of a localized 
integration by parts formula. As a result of such an explicit calculation one obtains the Taylor expansion of the density in Theorem 3.11. In particular we obtain explicit expressions for the constants in the expansion. This allows us to prove that the terms with odd powers vanish.

For the expansion to hold we assume hypotheses (H1)-(H3) which are natural to the problem at hand. (H4) is a technical condition. At the end of Section 3 , as an example, we give a class of coefficients that satisfy this condition. Finally, Section 4 is an appendix which contains technical results.

We refer the reader to [Nualart (1995)] and [Nualart (1998)] for all notions and notations on Malliavin Calculus used along the paper. As usually we denote by $C$ any real constant, independently of its values.

\section{Preliminary results}

This section is devoted to state some properties of the solution of the evolution equation (2.1) which are needed in the forthcoming sections.

We introduce some hypothesis on the coefficients and the initial condition:

(H1) $\sigma, b: \mathbb{R} \longrightarrow \mathbb{R}$ are $\mathcal{C}^{\infty}$-functions with bounded derivatives of any order greater than 1 , and $X_{0} \in \mathcal{C}([0,1])$.

(H2) There exists $C>0$ such that $\inf \{|\sigma(y)|, y \in \mathbb{R}\} \geq C$.

Set $X^{\varepsilon, h}(t, x)(\omega)=X^{\varepsilon}(t, x)\left(\omega+\frac{h}{\varepsilon}\right), \varepsilon \in(0,1],(t, x) \in[0, T] \times[0,1], h \in \mathcal{H}$.

The process $\left\{X^{\varepsilon, h}(t, x),(t, x) \in[0, T] \times[0,1]\right\}$ satisfies the equation

$$
\begin{gathered}
X^{\varepsilon, h}(t, x)=\int_{0}^{1} G_{t}(x, y) X_{0}(y) d y+\int_{0}^{t} \int_{0}^{1} G_{t-s}(x, y)\left\{\varepsilon \sigma\left(X^{\varepsilon, h}(s, y)\right)\right. \\
\left.\times W(d s, d y)+\sigma\left(X^{\varepsilon, h}(s, y)\right) \dot{h}(s, y) d s d y+b\left(X^{\varepsilon, h}(s, y)\right) d s d y\right\},
\end{gathered}
$$

and, by uniqueness of solution, $X^{\varepsilon, 0}(t, x)=X^{\varepsilon}(t, x)$ and $X^{0, h}(t, x)=$ $\Psi_{X_{0}}^{h}(t, x)$, where $X^{0, h}(t, x)=\lim _{\varepsilon \downarrow 0} X^{\varepsilon, h}(t, x)$ (see Proposition 2.1).

In the sequel $j$ will denote a positive integer. Let $X_{j}^{\varepsilon, h}(t, x), j \geq 1, \varepsilon \in[0,1]$, 
be the solutions of the following stochastic differential equations:

$$
\begin{aligned}
& X_{1}^{\varepsilon, h}(t, x)=\int_{0}^{t} \int_{0}^{1} G_{t-s}(x, y)\left\{\sigma\left(X^{\varepsilon, h}(s, y)\right) W(d s, d y)+\varepsilon \sigma^{\prime}\left(X^{\varepsilon, h}(s, y)\right)\right. \\
& \quad \times X_{1}^{\varepsilon, h}(s, y) W(d s, d y)+\sigma^{\prime}\left(X^{\varepsilon, h}(s, y)\right) X_{1}^{\varepsilon, h}(s, y) \dot{h}(s, y) d s d y \\
& \left.\quad+b^{\prime}\left(X^{\varepsilon, h}(s, y)\right) X_{1}^{\varepsilon, h}(s, y) d s d y\right\}
\end{aligned}
$$

and, for $j \geq 2$,

$$
\begin{aligned}
& X_{j}^{\varepsilon, h}(t, x)=I_{j-1}^{\varepsilon, h}(t, x)+\int_{0}^{t} \int_{0}^{1} G_{t-s}(x, y)\left\{\varepsilon \sigma^{\prime}\left(X^{\varepsilon, h}(s, y)\right) X_{j}^{\varepsilon, h}(s, y)\right. \\
& \quad \times W(d s, d y)+\sigma^{\prime}\left(X^{\varepsilon, h}(s, y)\right) X_{j}^{\varepsilon, h}(s, y) h(s, y) d s d y \\
& \left.\quad+b^{\prime}\left(X^{\varepsilon, h}(s, y)\right) X_{j}^{\varepsilon, h}(s, y) d s d y\right\},
\end{aligned}
$$

where

$$
\begin{aligned}
& I_{j-1}^{\varepsilon, h}(t, x)=\int_{0}^{t} \int_{0}^{1} G_{t-s}(x, y)\left\{\sum_{k=1}^{j-1} \sum_{\substack{\beta_{1}+\ldots+\beta_{k}=j-1 \\
\beta_{1}, \ldots, \beta_{k} \geq 1}} d_{j-1}\left(\beta_{1}, \ldots, \beta_{k}\right)\right. \\
& \times \sigma^{(k)}\left(X^{\varepsilon, h}(s, y)\right) \prod_{\ell=1}^{k} X_{\beta_{\ell}}^{\varepsilon, h}(s, y) W(d s d y)+\sum_{k=2}^{j} \sum_{\substack{\beta_{1}+\ldots+\beta_{k}=j \\
\beta_{1}, \ldots, \beta_{k} \geq 1}} c_{j}\left(\beta_{1}, \ldots, \beta_{k}\right) \\
& \times\left[\varepsilon \sigma^{(k)}\left(X^{\varepsilon, h}(s, y)\right) \prod_{\ell=1}^{k} X_{\beta_{\ell}}^{\varepsilon, h}(s, y)\left[W(d s, d y)+\frac{\dot{h}(s, y)}{\varepsilon} d s d y\right]\right. \\
& \left.\left.+b^{(k)}\left(X^{\varepsilon, h}(s, y)\right) \prod_{\ell=1}^{k} X_{\beta_{\ell}}^{\varepsilon, h}(s, y) d s d y\right]\right\}
\end{aligned}
$$

the coefficients $c_{j}\left(\beta_{1}, \ldots, \beta_{k}\right)$ and $d_{j}\left(\beta_{1}, \ldots, \beta_{k}\right)$ are obtained by induction. In particular, when $j=2, c_{2}(1,1)=1, d_{1}(1)=2$, and for $\varepsilon=0$,

$$
\begin{aligned}
& I_{1}^{0, h}(t, x)=\int_{0}^{t} \int_{0}^{1} G_{t-s}(x, y)\left\{2 \sigma^{\prime}\left(\Psi_{X_{0}}^{h}(s, y)\right) X_{1}^{0, h}(s, y) W(d s d y)\right. \\
& \quad+\sigma^{\prime \prime}\left(\Psi_{X_{0}}^{h}(s, y)\right) X_{1}^{0, h}(s, y)^{2} \dot{h}(s, y) d s d y \\
& \left.\quad+b^{\prime \prime}\left(\Psi_{X_{0}}^{h}(s, y)\right) X_{1}^{0, h}(s, y)^{2} d s d y\right\} .
\end{aligned}
$$


We use the conventions $X_{0}^{\varepsilon, h}(t, x)=X^{\varepsilon, h}(t, x)$ and $X_{0}^{0, h}(t, x)=\Psi_{X_{0}}^{h}(t, x)$. Equation (2.1) has a similar structure than (1.2), with the new term involving $h$. This novelty does not rise up any technical problem. For this reason, some results on the paths of $X^{\varepsilon}(t, x)$ that have been proved in [Márquez-Carreras and Sanz-Solé (1998b)] can be extended to the solution of (2.1), using the same arguments.

We now give the precise statements and the reference of the corresponding results for $X^{\varepsilon}(t, x)$, from which the proof can be borrowed.

Proposition 2.1 Assume $(\mathrm{H} 1)$. Fix $(t, x) \in(0, T] \times(0,1], h \in \mathcal{H}$. Then there exists a version of $\left\{X^{\varepsilon, h}(t, x), \varepsilon \in(0,1)\right\}$ which is a $\mathcal{C}^{\infty}$ function with respect to $\varepsilon$ and, for any $j \geq 1$

$$
\frac{d^{j} X^{\varepsilon, h}}{d \varepsilon^{j}}(t, x)=X_{j}^{\varepsilon, h}(t, x) .
$$

Furthermore, for any $j \geq 0, p \in(1, \infty), \varepsilon, \varepsilon^{\prime} \in(0,1)$,

$$
\sup _{t, x} E\left(\left|X_{j}^{\varepsilon, h}(t, x)-X_{j}^{\varepsilon^{\prime}, h}(t, x)\right|^{p}\right) \leq C\left|\varepsilon-\varepsilon^{\prime}\right|^{p} .
$$

Consequently,

$$
E\left\{\sup _{0 \leq \varepsilon \leq 1}\left|X_{j}^{\varepsilon, h}(t, x)\right|^{p}\right\}<\infty
$$

Moreover,

$$
\lim _{\varepsilon \downarrow 0} X_{j}^{\varepsilon, h}(t, x)=X_{j}^{0, h}(t, x), \text { a.s. }
$$

See [Márquez-Carreras and Sanz-Solé (1998b)], Proposition 3.1 and Corollary 3.2 .

For any $\varepsilon \in(0,1]$ set

$$
\begin{aligned}
\hat{X}^{\varepsilon, h}(t, x) & =\frac{X^{\varepsilon, h}(t, x)-\Psi_{X_{0}}^{h}(t, x)}{\varepsilon}, \\
S^{\varepsilon, h}(t, x) & =\frac{X^{\varepsilon, h}(t, x)-\Psi_{X_{0}}^{h}(t, x)-\varepsilon X_{1}^{0, h}(t, x)}{\varepsilon^{2}} .
\end{aligned}
$$

Notice that

$$
S^{\varepsilon, h}(t, x)=\frac{\hat{X}^{\varepsilon, h}(t, x)-X_{1}^{0, h}(t, x)}{\varepsilon} .
$$

The functions $\hat{X}^{\varepsilon, h}(t, x)$ and $S^{\varepsilon, h}(t, x)$ are clearly $\mathcal{C}^{\infty}$ with respect to $\varepsilon \in$ $(0,1)$. The next Lemma states the relationship between the derivatives 
of the processes $X^{\varepsilon, h}(t, x), \hat{X}^{\varepsilon, h}(t, x)$ and $S^{\varepsilon, h}(t, x)$. For any $j \geq 1$ set $\hat{X}_{j}^{\varepsilon, h}(t, x)=\frac{d^{j} \hat{X}^{\varepsilon, h}(t, x)}{d \varepsilon^{j}}$ and $S_{j}^{\varepsilon, h}(t, x)=\frac{d^{j} S^{\varepsilon, h}(t, x)}{d \varepsilon^{j}}$.

As before we use the conventions $\hat{X}_{0}^{\varepsilon, h}(t, x)=\hat{X}^{\varepsilon, h}(t, x)$ and $S_{0}^{\varepsilon, h}(t, x)=$ $S^{\varepsilon, h}(t, x)$.

Lemma 2.2 Assume (H1). Then, for any $j \geq 0, \varepsilon \in(0,1)$,

$$
\begin{gathered}
\hat{X}_{j}^{\varepsilon, h}(t, x)=\frac{1}{j+1}\left\{X_{j+1}^{0, h}(t, x)+\varepsilon \int_{0}^{1}\left(1-\xi^{j+1}\right) X_{j+2}^{\varepsilon \xi, h}(t, x) d \xi\right\} \\
S_{j}^{\varepsilon, h}(t, x)=\frac{1}{(j+1)(j+2)} X_{j+2}^{0, h}(t, x)+\frac{\varepsilon}{(j+1)} \int_{0}^{1}\left(1-\xi^{j+1}\right) \hat{X}_{j+2}^{\varepsilon \xi, h}(t, x) d \xi .
\end{gathered}
$$

See [Márquez-Carreras and Sanz-Solé (1998b)], Lemma 3.3.

Clearly, (2.5), (2.9) and (2.10) yield, for any $j \geq 0$,

$$
\begin{aligned}
\hat{X}_{j}^{0, h}(t, x) & :=\lim _{\varepsilon \downarrow 0} \hat{X}_{j}^{\varepsilon, h}(t, x)=\frac{1}{j+1} X_{j+1}^{0, h}(t, x), \\
S_{j}^{0, h}(t, x) & :=\lim _{\varepsilon \downarrow 0} S_{j}^{\varepsilon, h}(t, x)=\frac{1}{(j+1)(j+2)} X_{j+2}^{0, h}(t, x) .
\end{aligned}
$$

By the mean value theorem, one can easily check that the processes $\left\{\hat{X}^{\varepsilon, h}(t, x) ;(t, x) \in[0, T] \times[0,1]\right\},\left\{S^{\varepsilon, h}(t, x) ;(t, x) \in[0, T] \times[0,1]\right\}$ given in (2.6) and (2.7), respectively, satisfy the following equations

$$
\begin{aligned}
\hat{X}^{\varepsilon, h}(t, x)= & \int_{0}^{t} \int_{0}^{1} G_{t-s}(x, y) \sigma\left(X^{\varepsilon, h}(s, y)\right) W(d s, d y) \\
& +\int_{0}^{t} \int_{0}^{1} G_{t-s}(x, y)\left\{\partial \sigma^{\varepsilon}(s, y) \hat{X}^{\varepsilon, h}(s, y) \dot{h}(s, y)\right. \\
& \left.+\partial b^{\varepsilon}(s, y) \hat{X}^{\varepsilon, h}(s, y)\right\} d s d y, \\
S^{\varepsilon, h}(t, x)= & \int_{0}^{t} \int_{0}^{1} G_{t-s}(x, y) \partial \sigma^{\varepsilon}(s, y) \hat{X}^{\varepsilon, h}(s, y) W(d s, d y) \\
& +\int_{0}^{t} \int_{0}^{1} G_{t-s}(x, y)\left\{\sigma^{\prime}\left(\Psi_{X_{0}}^{h}(s, y)\right) S^{\varepsilon, h}(s, y) \dot{h}(s, y)\right. \\
& +\partial^{2} \sigma^{\varepsilon}(s, y) \hat{X}^{\varepsilon, h}(s, y)^{2} \dot{h}(s, y)+b^{\prime}\left(\Psi_{X_{0}}^{h}(s, y)\right) S^{\varepsilon, h}(s, y) \\
& \left.+\partial^{2} b^{\varepsilon}(s, y) \hat{X}^{\varepsilon, h}(s, y)^{2}\right\} d s d y,
\end{aligned}
$$


with $\partial \sigma^{\varepsilon}(s, y)=\int_{0}^{1} \sigma^{\prime}\left(\Psi_{X_{0}}^{h}(s, y)+\lambda\left(X^{\varepsilon, h}(s, y)-\Psi_{X_{0}}^{h}(s, y)\right)\right) d \lambda, \partial^{2} \sigma^{\varepsilon}(s, y)$ $=\int_{0}^{1} d u \int_{0}^{u} d v \sigma^{\prime \prime}\left(\Psi_{X_{0}}^{h}(s, y)+v\left(X^{\varepsilon, h}(s, y)-\Psi_{X_{0}}^{h}(s, y)\right)\right)$ and a similar definition for $\partial b^{\varepsilon}(s, y), \partial^{2} b^{\varepsilon}(s, y)$, respectively. From (2.12), (2.3) and (2.4) we see that $S^{0, h}(t, x)$ satisfies

$$
\begin{aligned}
S^{0, h}(t, x)= & \int_{0}^{t} \int_{0}^{1} G_{t-s}(x, y) \sigma^{\prime}\left(\Psi_{X_{0}}^{h}(s, y)\right) X_{1}^{0, h}(s, y) W(d s, d y) \\
& +\int_{0}^{t} \int_{0}^{1} G_{t-s}(x, y)\left\{\sigma^{\prime}\left(\Psi_{X_{0}}^{h}(s, y)\right) S^{0, h}(s, y) \dot{h}(s, y)\right. \\
& +\frac{1}{2} \sigma^{\prime \prime}\left(\Psi_{X_{0}}^{h}(s, y)\right) X_{1}^{0, h}(s, y)^{2} \dot{h}(s, y)+b^{\prime}\left(\Psi_{X_{0}}^{h}(s, y)\right) S^{0, h}(s, y) \\
& \left.+\frac{1}{2} b^{\prime \prime}\left(\Psi_{X_{0}}^{h}(s, y)\right) X_{1}^{0, h}(s, y)^{2}\right\} d s d y .
\end{aligned}
$$

Assume (H1). Then the standard arguments developed, for instance, in [Bally and Pardoux (1998)] yield that for any fixed $(t, x) \in(0, T] \times(0,1)$, $\hat{X}_{j}^{\varepsilon, h}(t, x), \quad S_{j}^{\varepsilon, h}(t, x)$ belong to $\mathbb{D}^{\infty}$, for any $j \geq 0$. Moreover, for any $j \in \mathbb{Z}_{+}, k \in \mathbb{N}, p \in(0,1)$,

$$
\begin{aligned}
& \sup _{0<\varepsilon \leq 1} \sup _{t, x}\left\|\hat{X}_{j}^{\varepsilon, h}(t, x)\right\|_{k, p} \leq C, \\
& \sup _{0<\varepsilon \leq 1} \sup _{t, x}\left\|S_{j}^{\varepsilon, h}(t, x)\right\|_{k, p} \leq C,
\end{aligned}
$$

where $\|\cdot\|_{k, p}$ denotes the norm of the Sobolev space $\mathbb{D}^{k, p}$ (see [Nualart (1995)] Section 1.5 for the definitions of $\mathbb{D}^{k, p},\|\cdot\|_{k, p}$ and $\left.\mathbb{D}^{\infty}\right)$.

If, in addition, (H2) holds, then (see [Millet and Sanz-Solé (1996)])

$$
\sup _{0<\varepsilon \leq 1} E\left(\left|\operatorname{det}, \hat{X}_{\hat{X}, h(t, x)}^{-1}\right|^{p}\right)<C, \quad p \in(1, \infty),
$$

and $X_{1}^{0, h}(t, x)$ is a nondegenerate Gaussian random variable, where, $\hat{X}^{\epsilon, h}(t, x)$ denotes the Malliavin matrix of $\hat{X}^{\varepsilon, h}(t, x)$ (see [Nualart (1995)] pg. 81).

\section{Taylor expansion of the density}

In this section we consider a fixed $(t, x) \in(0, T] \times(0,1], y \in \mathbb{R}, y \neq \Psi_{X_{0}}^{0}(t, x)$ and we establish a Taylor expansion for the density $p_{t, x}^{\varepsilon}(y)$ of $X^{\varepsilon}(t, x)$ at $y$. 
As in [Watanabe (1987)] the proof uses a localization procedure based on an additional hypothesis (see (H3) below).

Let $K_{y}=\left\{h \in \mathcal{H}: \Psi_{X_{0}}^{h}(t, x)=y\right\}$ and $K_{y}^{\text {min }}$ the set of those $h \in K_{y}$ that minimize the $\mathcal{H}$-norm. In [Millet and Sanz-Solé (1996)] we have proved that (H1) and (H2) yield $K_{y}^{\min } \neq \emptyset$.

We now introduce an additional condition, as follows:

(H3) The set $K_{y}^{\min }$ is finite.

Set $K_{y}^{\min }=\left\{h_{1}, \ldots, h_{n_{0}}\right\}$ and $a=\left\|h_{i}\right\|_{\mathcal{H}}^{2}, i=1, \ldots, n_{0}$.

Consider a family $\left\{\phi_{\rho}, \rho \in(0,1]\right\}$ of $\mathcal{C}^{\infty}$-functions on $\mathbb{R}$, increasing with $\rho$, such that $\sup _{0<p \leq 1}\left\|\phi_{\rho}\right\|_{\infty} \leq 1, \phi_{\rho}(x)=0$ if $|x|>\rho$ and $\phi_{\rho}(x)=1$ if $|x| \leq \frac{\rho}{2}$. Fix $\beta \in(2, \infty)$ and define, for any $\rho \in(0,1], \varepsilon>0$,

$$
\chi_{\rho, \beta}(\varepsilon, \omega)=\sum_{i=1}^{n_{0}} \phi_{\rho}\left(\left\|X^{\varepsilon}(\omega)-\Psi_{X_{0}}^{h_{i}}\right\|_{L^{\beta}([0, T] \times[0,1])}^{\beta}\right) .
$$

Then,

$$
p_{t, x}^{\varepsilon}(y)=E\left(\delta_{\{y\}}\left(X^{\varepsilon}(t, x)\right)\right)=p_{t, x}^{\varepsilon, 1}(y)+p_{t, x}^{\varepsilon, 2}(y),
$$

where $\delta_{\{y\}}$ denotes the Dirac $\delta$-function at $y$ and

$$
\begin{aligned}
& p_{t, x}^{\varepsilon, 1}(y)=E\left\{\left(1-\chi_{\rho, \beta}(\varepsilon, \omega)\right) \delta_{\{y\}}\left(X^{\varepsilon}(t, x)\right)\right\}, \\
& p_{t, x}^{\varepsilon, 2}(y)=E\left\{\chi_{\rho, \beta}(\varepsilon, \omega) \delta_{\{y\}}\left(X^{\varepsilon}(t, x)\right)\right\} .
\end{aligned}
$$

Denote by $B^{\beta}(x, \rho)$ the open ball in $L^{\beta}([0, T] \times[0,1])$ centered at $x$, with radius $\rho$. Under $(\mathrm{H} 2)$ there exists $\rho_{0}>0$ such that for any $\rho \in\left(0, \rho_{0}\right)$ the balls $B^{\beta}\left(\Psi_{X_{0}}^{h_{i}}, \rho\right), i=1, \ldots, n_{0}$, are pairwise disjoint.

Our first purpose is to study $p_{t, x}^{\varepsilon, 1}(y)$.

Proposition 3.1 Assume (H1)-(H3). For every $\rho \in\left(0, \rho_{0}\right)$ there exists a constant $C>0$ such that

$p_{t, x}^{\varepsilon, 1}(y)=E\left\{\left(1-\chi_{\rho, \beta}(\varepsilon, \omega)\right) \delta_{\{y\}}\left(X^{\varepsilon}(t, x)\right)\right\}=O\left(\exp \left\{-\frac{1}{2 \varepsilon^{2}}(a+C)\right\}\right)$,

as $\varepsilon \downarrow 0$. 
Proof: Let $\rho^{\prime}>0$ and set $\Psi=\left(1-\chi_{\rho, \beta}(\varepsilon, \omega)\right) \phi_{\rho^{\prime}}\left(X^{\varepsilon}(t, x)-y\right)$. The integration by parts formula of the Malliavin Calculus and the local property of the Skorohod integral yield

$$
\begin{aligned}
& 0 \leq E\left\{\left(1-\chi_{\rho, \beta}(\varepsilon, \omega)\right) \delta_{\{y\}}\left(X^{\varepsilon}(t, x)\right)\right\}=E\left\{\Psi \delta_{\{y\}}\left(X^{\varepsilon}(t, x)\right)\right\} \\
& \leq E\left\{1_{\left\{X^{\varepsilon}(t, x)>y\right\}} H_{1}\left(X^{\varepsilon}(t, x), \Psi\right)\right.
\end{aligned}
$$

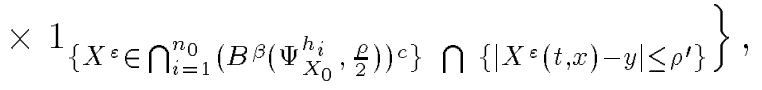

with $H_{1}\left(X^{\varepsilon}(t, x), \Psi\right)=\delta\left(\Psi\left\|D X^{\varepsilon}(t, x)\right\|^{-2} D X^{\varepsilon}(t, x)\right)$, where $\delta$ denotes the Skorohod integral (see [Nualart (1998)]).

Let ${ }_{\varepsilon}$ denote the Malliavin matrix of $X^{\varepsilon}(t, x)$. Lemma 2.4 in [Millet and Sanz-Solé (1996)] implies

$$
\left\|,{ }_{\varepsilon}^{-1}\right\|_{p} \leq C \varepsilon^{-2}, \quad p \geq 1 .
$$

Thus, by Hölder's inequality and the $L^{p}$-bounds for the Skorohod integral,

$$
E\left\{\left(1-\chi_{\rho, \beta}(\varepsilon, \omega)\right) \delta_{\{y\}}\left(X^{\varepsilon}(t, x)\right)\right\} \leq T_{1}^{1 / p} T_{2}^{1 / q}
$$

with $\frac{1}{p}+\frac{1}{q}=1$, and

$$
\begin{aligned}
& T_{1}=P\left\{X^{\varepsilon} \in \bigcap_{i=1}^{n_{0}}\left(B^{\beta}\left(\Psi_{X_{0}}^{h_{i}}, \frac{\rho}{2}\right)\right)^{c},\left|X^{\varepsilon}(t, x)-y\right| \leq \rho^{\prime}\right\}, \\
& T_{2}=\left\|H_{1}\left(X^{\varepsilon}(t, x), \Psi\right)\right\|_{q}^{q} \leq C\left\|,{ }_{\varepsilon}^{-1}\right\|_{r}^{a}\left\|X^{\varepsilon}(t, x)\right\|_{2, s}^{b}\|\Psi\|_{1, s^{\prime}}^{d},
\end{aligned}
$$

where $r, s, s^{\prime}, a, b$, and $d$ are some positive real numbers greater than 1 .

The estimate (3.3) together with (3.21) in [Márquez-Carreras and Sanz-Solé (1998b)] yield $T_{2} \leq C \varepsilon^{-2 a}$, for some $a>0$. Consequently, letting $p \rightarrow 1$,

$$
\begin{aligned}
& \limsup _{\varepsilon \downarrow 0} \varepsilon^{2} \log E\left(\left(1-\chi_{\rho, \beta}(\varepsilon, \omega)\right) \delta_{\{y\}}\left(X^{\varepsilon}(t, x)\right)\right) \\
& \quad \leq \limsup _{\varepsilon \downarrow 0} \varepsilon^{2} \log P\left\{X^{\varepsilon} \in \bigcap_{i=1}^{n_{0}}\left(B^{\beta}\left(\Psi_{X_{0}}^{h_{i}}, \frac{\rho}{2}\right)\right)^{c},\left|X^{\varepsilon}(t, x)-y\right| \leq \rho^{\prime}\right\} .
\end{aligned}
$$

Let $\varphi_{0}^{i} \in \mathcal{C}([0, T] \times[0,1]), i=1, \ldots, n_{0}, \gamma, \rho^{\prime}>0$ and $y \in \mathbb{R}$. The set $\left\{\varphi \in \mathcal{C}([0, T] \times[0,1]): \varphi \in \bigcap_{i=1}^{n_{0}}\left(B^{\beta}\left(\varphi_{0}^{i}, \gamma\right)\right)^{c} ;|\varphi(t, x)-y| \leq \rho^{\prime}\right\}$ is closed in the topology of the uniform norm. Therefore, the large deviation principle 
for the family $\left\{X^{\varepsilon}, \varepsilon \in(0,1]\right\}$ established in [Chenal and Millet (1997)] (see also [Sowers (1992)]), shows that the right hand-side of (3.4) is bounded by

$$
-\inf \left\{\frac{1}{2}\|h\|_{\mathcal{H}}^{2}: h \in \mathcal{H}, \Psi_{X_{0}}^{h} \in \bigcap_{i=1}^{n_{0}}\left(B^{\beta}\left(\Psi_{X_{0}}^{h_{i}}, \frac{\rho}{2}\right)\right)^{c},\left|\Psi_{X_{0}}^{h}(t, x)-y\right| \leq \rho^{\prime}\right\} .
$$

There exists $\rho^{\prime}>0$ such that this last quantity is strictly bounded by $-a / 2$. Indeed, otherwise we could choose $\rho^{\prime}=1 / n, h^{n} \in \mathcal{H}$ satisfying

$$
\Psi_{X_{0}}^{h^{n}} \in \bigcap_{i=1}^{n_{0}}\left(B^{\beta}\left(\Psi_{X_{0}}^{h_{i}}, \frac{\rho}{2}\right)\right)^{c},\left|\Psi_{X_{0}}^{h^{n}}(t, x)-y\right| \leq \frac{1}{n}
$$

and $\limsup \sup _{n}\left\|h^{n}\right\|_{\mathcal{H}}^{2} \leq a$. Let $\left\{h^{n_{k}}, h \geq 1\right\}$ be a subsequence of $\left\{h^{n}, n \geq 1\right\}$ converging weakly to some $\bar{h} \in \mathcal{H}$. Clearly, $\|\bar{h}\|_{\mathcal{H}}^{2} \leq a$ and, by the continuity property of the skeleton proved in [Chenal and Millet (1997)], $\Psi_{X_{0}}^{\bar{h}}(t, x)=y$ and

$$
\Psi_{X_{0}}^{\bar{h}} \in \bigcap_{i=1}^{n_{0}}\left(B^{\beta}\left(\Psi_{X_{0}}^{h_{i}}, \frac{\rho}{2}\right)\right)^{c} .
$$

Thus, by (H3), $\bar{h} \in K_{y}^{\min }$ and $\bar{h}=h_{i}$ for some $i=1, \ldots, n_{0}$. This leads to a contradiction with (3.5). Hence

$$
\limsup _{\varepsilon \downarrow 0} \varepsilon^{2} \log E\left(\left(1-\chi_{\rho, \beta}(\varepsilon, \omega)\right) \delta_{\{y\}}\left(X^{\varepsilon}(t, x)\right)\right)<-\frac{1}{2} a,
$$

proving (3.2).

Our next aim is to analyze the contribution of the term $p_{t, x}^{\varepsilon, 2}(y)$. Without loss of generality we will assume that $K_{y}^{\min }$ consists of a unique element, denoted by $\bar{h}$ in the sequel.

Consider the transformation of the Wiener space defined by $T^{\varepsilon, \bar{h}}(\omega)=\omega+\frac{\bar{h}}{\varepsilon}$; the Cameron-Martin-Girsanov theorem, Lemma 4.2 and the identity (2.8) yield

$$
\begin{aligned}
p_{t, x}^{\varepsilon, 2}(y)=E & \left\{\chi_{\rho, \beta}^{\bar{h}}(\varepsilon, \omega) \delta_{\{y\}}\left(X^{\varepsilon}(t, x)\right)\right\}=\frac{1}{\varepsilon} \exp \left(-\frac{1}{2 \varepsilon^{2}}\|\bar{h}\|_{\mathcal{H}}^{2}\right) \\
& \times E\left\{\exp \left(\bar{\lambda} S^{\varepsilon, \bar{h}}(t, x)\right) \chi_{\rho, \beta}^{\bar{h}}\left(\varepsilon, \omega+\frac{\bar{h}}{\varepsilon}\right) \delta_{\{0\}}\left(\hat{X}^{\varepsilon, \bar{h}}(t, x)\right)\right\},
\end{aligned}
$$

where $\chi_{\rho, \beta}^{\bar{h}}(\varepsilon, \omega)=\phi_{\rho}\left(\left\|X^{\varepsilon}-\Psi_{X_{0}}^{\bar{h}}\right\|_{L^{\beta}([0, T] \times[0,1])}^{\beta}\right)$ and $\bar{\lambda}=\lambda^{\bar{h}}$, where $\lambda^{\bar{h}}$ is defined in Lemma 4.2 . 
The remaining of this section is essentially devoted to give the Taylor expansion of the right hand-side of (3.6).

We now give some crucial ingredients of the proof. In the next Lemmas 3.2 to 3.4 and in Proposition $3.5, h$ is an arbitrary element of $\mathcal{H}$. Although we assume (H1) to obtain these results, the proofs still go through under weaker conditions. For any $\beta \in(1, \infty), \varepsilon \in(0,1], \rho \in(0,1]$, and $h \in \mathcal{H}$, set

$$
A^{\varepsilon, \beta, \rho}=\left\{\left\|X^{\varepsilon, h}-\Psi_{X_{0}}^{h}\right\|_{L^{2 \beta}([0, T] \times[0,1])}^{2 \beta} \leq \rho\right\} .
$$

Lemma 3.2 Assume (H1). There exist $r_{0}, C>0$ such that, for any $r \geq r_{0}$,

$$
\begin{gathered}
P\left\{\left\|X_{1}^{0, h}\right\|_{\infty}>r\right\} \leq \exp \left(-\frac{r^{2}}{C}\right), \\
\sup _{0<\varepsilon \leq 1} P\left\{\left\|\hat{X}^{\varepsilon, h}\right\|_{\infty}>r, A^{\varepsilon, \beta, \rho}\right\} \leq \exp \left(-\frac{r^{2}}{C}\right),
\end{gathered}
$$

for every $\beta \in(3, \infty), \rho \in(0,1]$.

Proof: Using a version of Gronwall's lemma given, for instance, in [Walsh (1986)] it is easy to check that

$$
\sup _{0 \leq t \leq T} \sup _{0 \leq x \leq 1}\left|\Psi_{X_{0}}^{h}(t, x)\right| \leq C .
$$

Consequently,

$$
\left\|\sigma\left(\Psi_{X_{0}}^{h}\right)\right\|_{\infty} \leq C .
$$

Consider the equation satisfied by $\left\{X_{1}^{0, h}(t, x),(t, x) \in[0, T] \times[0,1]\right\}$ (see $\left.(2.2)\right)$. Schwarz's inequality implies

$$
\begin{aligned}
& \left|X_{1}^{0, h}(t, x)\right|^{2} \leq C\left\{\left|\int_{0}^{t} \int_{0}^{1} G_{t-s}(x, y) \sigma\left(\Psi_{X_{0}}^{h}(s, y)\right) W(d s, d y)\right|^{2}+\left(1+\|h\|_{\mathcal{H}}^{2}\right)\right. \\
& \left.\quad \times \int_{0}^{t} \frac{1}{\sqrt{t-s}} \sup _{0 \leq y \leq 1}\left|X_{1}^{0, h}(s, y)\right|^{2} d s\right\} .
\end{aligned}
$$

Then, using the above-mentioned Gronwall's lemma, we obtain

$$
\begin{aligned}
& \sup _{0 \leq t \leq T} \sup _{0 \leq x \leq 1}\left|X_{1}^{0, h}(t, x)\right| \\
& \quad \leq C \sup _{0 \leq t \leq T} \sup _{0 \leq x \leq 1}\left|\int_{0}^{t} \int_{0}^{1} G_{t-s}(x, y) \sigma\left(\Psi_{X_{0}}^{h}(s, y)\right) W(d s, d y)\right| .
\end{aligned}
$$


Therefore, by Lemma 4.1 and (3.10)

$$
\begin{aligned}
& P\left\{\left\|X_{1}^{0, h}\right\|_{\infty}>r\right\} \leq P\left\{\left\|\int_{0} \int_{0}^{1} G_{-s}(*, y) \sigma\left(\Psi_{X_{0}}^{h}(s, y)\right) W(d s, d y)\right\|_{\infty}>\frac{r}{C},\right. \\
& \left.\quad \int_{0}^{T} \int_{0}^{1}\left(\sigma\left(\Psi_{X_{0}}^{h}(s, y)\right)\right)^{2 \beta} d s d y \leq C T\right\} \\
& \quad \leq \exp \left(-\frac{r^{2}}{C}\right)
\end{aligned}
$$

for $r$ big enough. This proves (3.7).

The proof of (3.8) is similar. Again, the equation satisfied by $\left\{\hat{X}^{\varepsilon, h}(t, x)\right.$, $(t, x) \in[0, T] \times[0,1]\}$ and an argument based on Gronwall's lemma yield

$$
\left\|\hat{X}^{\varepsilon, h}\right\|_{\infty} \leq C\left\|\int_{0} \int_{0}^{1} G_{.-s}(*, y) \sigma\left(X^{\varepsilon, h}(s, y)\right) W(d s, d y)\right\|_{\infty} .
$$

The Lipschitz property of $\sigma$ and (3.10) imply that, on the set $A^{\varepsilon, \beta, \rho}$,

$$
\int_{0}^{T} \int_{0}^{1}\left|\sigma\left(X^{\varepsilon, h}(s, y)\right)\right|^{2 \beta} d s d y \leq R
$$

for some positive constant $R$ not depending on $\rho$.

Therefore, Lemma 4.1 applied to $\tau(s, y)=\sigma\left(X^{\varepsilon, h}(s, y)\right)$ yields (3.8).

Lemma 3.3 Assume (H1). For any $\rho \in(0,1], \beta \in(3, \infty)$, there exist $r_{0}, C$, not depending on $\rho$, such that, for every $r \geq r_{0}$,

$$
\sup _{0<\varepsilon \leq 1} P\left\{\left\|\hat{X}^{\varepsilon, h}-X_{1}^{0, h}\right\|_{\infty}>r, \quad A^{\varepsilon, \beta, \rho}\right\} \leq \exp \left(-\frac{r^{2}}{\rho^{1 / \beta}} C\right) .
$$

Proof. We recall that $\left(\hat{X}^{\varepsilon, h}-X_{1}^{0, h}\right)(t, x)=\varepsilon S^{\varepsilon, h}(t, x)$, where $\left\{S^{\varepsilon, h}(t, x)\right.$, $(t, x) \in[0, T] \times[0,1]\}$ satisfies $(2.14)$. More exactly, we have

$$
\begin{aligned}
& \left(\hat{X}^{\varepsilon, h}-X_{1}^{0, h}\right)(t, x)=\int_{0}^{t} \int_{0}^{1} G_{t-s}(x, y) \partial \sigma^{\varepsilon}(s, y)\left(X^{\varepsilon, h}-\Psi_{X_{0}}^{h}\right)(s, y) \\
& \quad \times W(d s, d y)+\int_{0}^{t} \int_{0}^{1} G_{t-s}(x, y)\left\{\left(\sigma^{\prime}\left(\Psi_{X_{0}}^{h}(s, y)\right) \dot{h}(s, y)+b^{\prime}\left(\Psi_{X_{0}}^{h}(s, y)\right)\right)\right. \\
& \quad \times\left(\hat{X}^{\varepsilon, h}-X_{1}^{0, h}\right)(s, y)+\left(\partial^{2} \sigma^{\varepsilon}(s, y) \dot{h}(s, y)+\partial^{2} b^{\varepsilon}(s, y)\right) \hat{X}^{\varepsilon, h}(s, y) \\
& \left.\quad \times\left(X^{\varepsilon, h}-\Psi_{X_{0}}^{h}\right)(s, y)\right\} d s d y .
\end{aligned}
$$


Therefore

$$
\begin{gathered}
\left|\left(\hat{X}^{\varepsilon, h}-X_{1}^{0, h}\right)(t, x)\right|^{2} \leq C\left\{\mid \int_{0}^{t} \int_{0}^{1} G_{t-s}(x, y) \partial \sigma^{\varepsilon}(s, y)\left(X^{\varepsilon, h}-\Psi_{X_{0}}^{h}\right)(s, y)\right. \\
\quad \times\left. W(d s d y)\right|^{2}+\int_{0}^{t} \frac{1}{(t-s)^{1 / 2}} \sup _{0 \leq x \leq 1}\left|\left(\hat{X}^{\varepsilon, h}-X_{1}^{0, h}\right)(s, x)\right|^{2} d s \\
\left.\quad+\int_{0}^{t} \int_{0}^{1} G_{t-s}^{2}(x, y)\left(\hat{X}^{\varepsilon, h}(s, y)\right)^{2}\left(\left(X^{\varepsilon, h}-\Psi_{X_{0}}^{h}\right)(s, y)\right)^{2} d s d y\right\} .
\end{gathered}
$$

Let $\alpha \in(1,3 / 2)$ satisfying $1 / \alpha+1 / \beta=1$. Hölder's inequality yields

$$
\begin{aligned}
& \int_{0}^{t} \int_{0}^{1} G_{t-s}^{2}(x, y)\left(\hat{X}^{\varepsilon, h}(s, y)\right)^{2}\left(\left(X^{\varepsilon, h}-\Psi_{X_{0}}^{h}\right)(s, y)\right)^{2} d s d y \\
& \quad \leq\left\|\hat{X}^{\varepsilon, h}\right\|_{\infty}^{2}\left(\int_{0}^{t} \int_{0}^{1} G_{t-s}^{2 \alpha}(x, y) d s d y\right)^{1 / \alpha}\left\|X^{\varepsilon, h}-\Psi_{X_{0}}^{h}\right\|_{L^{2 \beta}([0, T] \times[0,1])}^{2} \\
& \quad \leq C\left\|\hat{X}^{\varepsilon, h}\right\|_{\infty}^{2}\left\|X^{\varepsilon, h}-\Psi_{X_{0}}^{h}\right\|_{L^{2 \beta}([0, T] \times[0,1])}^{2}
\end{aligned}
$$

Substituting this estimate in (3.11) and using Gronwall's lemma we obtain

$$
P\left\{\left\|\hat{X}^{\varepsilon, h}-X_{1}^{0, h}\right\|_{\infty}>r, A^{\varepsilon, \beta, \rho}\right\} \leq p_{1}+p_{2},
$$

with

$$
\begin{aligned}
p_{1} & =P\left\{\left\|\hat{X}^{\varepsilon, h}\right\|_{\infty}>r_{1}, A^{\varepsilon, \beta, \rho}\right\} \\
p_{2} & =P\left\{\left\|\int_{0} \int_{0}^{1} G_{t-.}(*, y) \partial \sigma^{\varepsilon}(s, y)\left(X^{\varepsilon, h}-\Psi_{X_{0}}^{h}\right)(s, y) W(d s, d y)\right\|_{\infty}\right. \\
& \left.>\frac{r}{C_{2}}-r_{1} \rho^{\frac{1}{2 \beta}}, A^{\varepsilon, \beta, \rho}\right\}
\end{aligned}
$$

where $r_{1}=a r \rho^{-1 /(2 \beta)}$ with $1 / C_{2}-a>0$.

Then, the statement of the Lemma follows from Lemmas 3.2 and 4.1.

Lemma 3.4 Assume (H1). For any $\rho \in(0,1], \beta \in(3, \infty)$, there exist $r_{0}, C$, not depending on $\rho$, such that, for any $r \geq r_{0}$

$$
\sup _{0<\varepsilon \leq 1} P\left\{\left\|S^{\varepsilon, h}-S^{0, h}\right\|_{\infty}>r, A^{\varepsilon, \beta, \rho}\right\} \leq \exp \left(-\frac{r}{\rho^{\frac{1}{2 \beta}}} C\right) .
$$


Proof. From (2.14) and (2.15) it follows that the process $\left\{\left(S^{\varepsilon, h}-S^{0, h}\right)(t, x)\right.$, $(t, x) \in[0, T] \times[0,1]\}$ satisfies the equation.

$$
\begin{aligned}
& \left(S^{\varepsilon, h}-S^{0, h}\right)(t, x)=\int_{0}^{t} \int_{0}^{1} G_{t-s}(x, y)\left(\sigma^{\prime}\left(\Psi_{X_{0}}^{h}(s, y)\right) \dot{h}(s, y)+b^{\prime}\left(\Psi_{X_{0}}^{h}(s, y)\right)\right) \\
& \quad \times\left(S^{\varepsilon, h}-S^{0, h}\right)(s, y) d s d y+\sum_{i=1}^{4} T_{i}(t, x),
\end{aligned}
$$

with

$$
\begin{aligned}
T_{1}(t, x)= & \int_{0}^{t} \int_{0}^{1} G_{t-s}(x, y) \sigma^{\prime}\left(\Psi_{X_{0}}^{h}(s, y)\right)\left(\hat{X}^{\varepsilon, h}-X_{1}^{0, h}\right)(s, y) W(d s, d y), \\
T_{2}(t, x)= & \int_{0}^{t} \int_{0}^{1} G_{t-s}(x, y) \partial^{2} \sigma^{\varepsilon}(s, y) \hat{X}^{\varepsilon, h}(s, y)\left(X^{\varepsilon, h}-\Psi_{X_{0}}^{h}\right)(s, y) \\
& \quad \times W(d s, d y) \\
T_{3}(t, x)= & \int_{0}^{t} \int_{0}^{1} G_{t-s}(x, y) \dot{h}(s, y)\left(\partial^{2} \sigma^{\varepsilon}(s, y)\left(\hat{X}^{\varepsilon, h}(s, y)\right)^{2}\right. \\
& \left.\quad-\frac{1}{2} \sigma^{\prime \prime}\left(\Psi_{X_{0}}^{h}(s, y)\right)\left(X_{1}^{0, h}(s, y)\right)^{2}\right) d s d y, \\
T_{4}(t, x)= & \int_{0}^{t} \int_{0}^{1} G_{t-s}(x, y)\left(\partial^{2} b^{\varepsilon}(s, y)\left(\hat{X}^{\varepsilon, h}(s, y)\right)^{2}-\frac{1}{2} b^{\prime \prime}\left(\Psi_{X_{0}}^{h}(s, y)\right)\right. \\
& \left.\times\left(X_{1}^{0, h}(s, y)\right)^{2}\right) d s d y .
\end{aligned}
$$

For any $r, r_{i}, i=1,2,3$, positive real numbers, $\beta \in(3, \infty), \rho \in(0,1]$, set

$$
P\left\{\left\|S^{\varepsilon, h}-S^{0, h}\right\|_{\infty}>r, A^{\varepsilon, \beta, \rho}\right\} \leq \sum_{i=1}^{4} q_{i}^{\rho},
$$

where

$$
\begin{aligned}
q_{1}^{\rho}= & P\left\{\left\|S^{\varepsilon, h}-S^{0, h}\right\|_{\infty}>r, A^{\varepsilon, \beta, \rho},\left\|\hat{X}^{\varepsilon, h}-X_{1}^{0, h}\right\|_{\infty} \leq r_{1},\left\|\hat{X}^{\varepsilon, h}\right\|_{\infty} \leq r_{2},\right. \\
& \left.\left\|X_{1}^{0, h}\right\|_{\infty} \leq r_{3}\right\}, \\
q_{2}^{\rho}= & P\left\{\left\|\hat{X}^{\varepsilon, h}-S_{1}^{0, h}\right\|_{\infty}>r_{1}, A^{\varepsilon, \beta, \rho}\right\}, \\
q_{3}^{\rho}= & P\left\{\left\|\hat{X}^{\varepsilon, h}\right\|_{\infty}>r_{2}, A^{\varepsilon, \beta, \rho}\right\}, \\
q_{4}^{\rho}= & P\left\{\left\|X_{1}^{0, h}\right\|_{\infty}>r_{3}\right\} .
\end{aligned}
$$


Lemma 3.2 yields the existence of $r_{0}, C_{1}>0$ such that, for any $r_{2}, r_{3} \geq$ $r_{0}, \rho \in(0,1]$,

$$
q_{3}^{\rho}+q_{4}^{\rho} \leq \exp \left(-\frac{r_{2}^{2}}{C_{1}}\right)+\exp \left(-\frac{r_{3}^{2}}{C_{1}}\right)
$$

By Lemma 3.3, for any fixed $\rho \in(0,1]$, there exist $\bar{r}_{0}, C_{2}>0$ such that, for every $r \geq \bar{r}_{0}$,

$$
q_{2}^{\rho} \leq \exp \left(-\frac{r_{1}^{2}}{\rho^{1 / \beta}} C_{2}\right)
$$

Using (3.13) and the standard arguments based on Gronwall's lemma we obtain

$$
\left\|S^{\varepsilon, h}-S^{0, h}\right\|_{\infty} \leq C \sum_{i=1}^{4}\left\|T_{i}\right\|_{\infty} .
$$

Our next aim is to give upper bounds for $\left\|T_{i}\right\|_{\infty}, i=3,4$, on the set

$$
A_{r_{1} r_{2} r_{3}}^{\varepsilon, \beta, \rho}:=A^{\varepsilon, \beta, \rho} \cap\left\{\left\|\hat{X}^{\varepsilon, h}-X_{1}^{0, h}\right\|_{\infty} \leq r_{1},\left\|\hat{X}^{\varepsilon, h}\right\|_{\infty} \leq r_{2},\left\|X_{1}^{0, h}\right\|_{\infty} \leq r_{3}\right\} .
$$

Note that

$$
\begin{aligned}
& T_{3}(t, x)=\int_{0}^{t} \int_{0}^{1} G_{t-s}(x, y) \dot{h}(s, y)\left\{\partial^{3} \sigma^{\varepsilon}(s, y)\left(X^{\varepsilon, h}-\Psi_{X_{0}}^{h}\right)(s, y) \hat{X}^{\varepsilon, h}(s, y)^{2}\right. \\
& \quad+\frac{1}{2} \sigma^{\prime \prime}\left(\Psi_{X_{0}}^{h}(s, y)\right) \hat{X}^{\varepsilon, h}(s, y)\left(\hat{X}^{\varepsilon, h}-X_{1}^{0, h}\right)(s, y)+\frac{1}{2} \sigma^{\prime \prime}\left(\Psi_{X_{0}}^{h}(s, y)\right) \\
& \left.\quad \times X_{1}^{0, h}(s, y)\left(\hat{X}^{\varepsilon, h}-X_{1}^{0, h}\right)(s, y)\right\} d s d y,
\end{aligned}
$$

with $\partial^{3} \sigma^{\varepsilon}(s, y)=\int_{0}^{1} d u \int_{0}^{u} d v \int_{0}^{v} d w \sigma^{\prime \prime \prime}\left(\Psi_{X_{0}}^{h}(s, y)+w\left(X^{\varepsilon, h}-\Psi_{X_{0}}^{h}\right)(s, y)\right)$. Then, clearly, on $A_{r_{1} r_{2} r_{3}}^{\varepsilon, \beta, p}$,

$$
\left\|T_{3}\right\|_{\infty} \leq C\left(\rho^{\frac{1}{2 \beta}} r_{2}^{2}+r_{1} r_{2}+r_{1} r_{3}\right) .
$$

The same upper bound holds for $\left\|T_{4}\right\|_{\infty}$.

Thus, (3.17)-(3.19) yield, on the set $A_{r_{1} r_{2} r_{3}}^{\varepsilon, \beta, \rho}$

$$
\left\|S^{\varepsilon, h}-S^{0, h}\right\|_{\infty} \leq C\left(\rho^{\frac{1}{2 \beta}} r_{2}^{2}+r_{1} r_{2}+r_{1} r_{3}+\left\|T_{1}\right\|_{\infty}+\left\|T_{2}\right\|_{\infty}\right) .
$$

Let $a_{i} \in(0, \infty), i=1,2,3$, be such that $C_{0}:=\frac{1}{C}-a_{2}-\sqrt{a_{1} a_{2}}-\sqrt{a_{1} a_{3}}>0$, where $C$ is the positive constant appearing in the right hand-side of (3.20). Then, set

$$
r_{1}=\left(a_{1} r \rho^{1 / 2 \beta}\right)^{1 / 2}, r_{2}=\left(a_{2} r \rho^{-1 / 2 \beta}\right)^{1 / 2}, r_{3}=\left(a_{3} r \rho^{-1 / 2 \beta}\right)^{1 / 2} .
$$


Clearly, $q_{1}^{\rho} \leq q_{11}^{\rho}+q_{12}^{\rho}$, with

$$
\begin{aligned}
& q_{11}^{\rho}=P\left\{\left\|T_{1}\right\|_{\infty}>\frac{1}{2} C_{0} r, A_{r_{1} r_{2} r_{3}}^{\varepsilon, \beta, \rho},\right. \\
& q_{12}^{\rho}=P\left\{\left\|T_{2}\right\|_{\infty}>\frac{1}{2} C_{0} r, A_{r_{1} r_{2} r_{3}}^{\varepsilon, \beta, \rho} .\right.
\end{aligned}
$$

Lemma 4.1 applied to the process $\tau(s, y)=\sigma^{\prime}\left(\Psi_{X_{0}}^{h}(s, y)\right)\left(\hat{X}^{\varepsilon, h}-X_{1}^{0, h}\right)(s, y)$ yields the existence of positive constants $K_{1}, K_{2}$ such that,

$$
q_{11}^{\rho} \leq \exp \left(-\frac{r^{2} C_{0}^{2}}{4 T^{1 / \beta}\left\|\sigma^{\prime}\right\|_{\infty}^{2} r_{1}^{2}} K_{2}\right),
$$

whenever $r / r_{1}>K_{1}$.

Analogously, the same Lemma applied to $\tau(s, y)=\partial^{2} \sigma^{\varepsilon}(s, y) \hat{X}^{\varepsilon, h}(s, y)$ $\left(X^{\varepsilon, h}-\Psi_{X_{0}}^{h}\right)(s, y)$, ensures the existence of positive constants $K_{1}, K_{2}$ such that

$$
q_{12}^{\rho} \leq \exp \left(-\frac{r^{2} C_{0}^{2}}{4\left\|\sigma^{\prime \prime}\right\|_{\infty}^{2} r_{2}^{2} \rho^{1 / \beta}} K_{2}\right)
$$

for $\frac{r}{r_{2} \rho^{1 / 2 \beta}} \geq K_{1}$.

Substituting the values of $r_{i}, i=1,2,3$, given in (3.21) into the inequalities (3.15), (3.16), (3.22) and (3.23) and, taking into account (3.14), we finish the proof of the Lemma.

Proposition 3.5 Assume (H1). For any $p>0, \beta \in(6, \infty)$, there exists $\rho_{1}=\rho_{1}(p) \in(0,1]$ such that

$$
\sup _{0<\varepsilon \leq 1} E\left\{\exp \left(p\left|\left(S^{\varepsilon, h}-S^{0, h}\right)(t, x)\right|\right) \chi_{\rho_{1}, \beta}^{h}\left(\varepsilon, \omega+\frac{h}{\varepsilon}\right)\right\}<+\infty .
$$

Proof. Fix $p>0, \beta>6$. Let $r_{0}, C$ be such that (3.12) is satisfied for any $r \geq r_{0}$; then choose $\rho_{1} \in(0,1]$ such that $p<C / \rho_{1}^{\beta / 2}$. Clearly $\chi_{\rho_{1}, \beta}^{h}(\varepsilon, \omega+$ $\left.\frac{h}{\varepsilon}\right) \leq 1_{A^{\varepsilon, \beta / 2, \rho_{1}}}$. Thus Fubini's theorem and the choice of $\rho_{1}$ yield

$$
\begin{aligned}
& E\left\{\exp \left(p\left|\left(S^{\varepsilon, h}-S^{0, h}\right)(t, x)\right|\right) \chi_{\rho_{1}, \beta}^{h}\left(\varepsilon, \omega+\frac{h}{\varepsilon}\right)\right\} \\
& \leq E\left\{\exp \left(p\left|\left(S^{\varepsilon, h}-S^{0, h}\right)(t, x)\right|\right) 1_{\left.A^{\varepsilon, \frac{\beta}{2}, \rho_{1}}\right\}}\right\} \\
& \leq e^{p r_{0}}+E\left\{\left(\int_{r_{0}}^{\left|S^{\varepsilon, h}-S^{0, h}\right|(t, x)} p e^{p y} d y\right) 1_{\left.A^{\varepsilon, \frac{\beta}{2}, \rho_{1}}\right\}}\right\} \\
& \leq C+p \int_{r_{0}}^{\infty} e^{-y\left(\frac{C}{\rho_{1}^{1 / 2 \beta}}-p\right)} d y<+\infty .
\end{aligned}
$$


The preceding Proposition is one of the ingredients used in the proof of the integration by parts formula well-suited to our problem proved in Proposition 3.8. The additional ingredient, stated in Proposition 3.6, needs a new requirement (see $(\mathrm{H} 4)$ below).

The random variable $S^{0, h}(t, x)$ has only components in the Wiener-Chaos of order 0 and 2. Indeed, from (2.15) one easily checks that the second order Malliavin derivative is deterministic and, in addition, $E\left(D S^{0, h}(t, x)\right)=0$. Set $Y(t, x)=\lambda^{h} S^{0, h}(t, x), h \in K_{y}^{\mathrm{min}}$, where $\lambda^{h}$ is defined in Lemma 4.2. Then

$$
\begin{aligned}
Y(t, x)=E( & Y(t, x))+\int_{[0, t] \times[0,1]} \int_{[0, t] \times[0,1]} f_{2}^{h}((t, x) ;(s, y),(r, z)) \\
& \times W(d s, d y) W(d r, d z)
\end{aligned}
$$

with $f_{2}^{h}((t, x) ; \cdot) \in L^{2}\left(([0, t] \times[0,1])^{2}\right)$. So, the kernel $f_{2}^{h}((t, x) ; \cdot)$ defines a Hilbert-Schmidt operator $A(t, x)$ on $H=L^{2}([0, t] \times[0,1])$. Denote by $\left\{\mu_{k}^{h}, k \geq 1\right\}$ the sequence of eigenvalues of this operator. Then

$$
Z(t, x)=Y(t, x)-E(Y(t, x))=\sum_{k=1}^{\infty} \mu_{k}^{h} \xi_{k} \otimes \xi_{k},
$$

with $\left\{\xi_{k}\right\}$ a CONS of the first Wiener-Chaos.

Following Proposition 8.4 in [Neveu (1968)], for any $\sigma>0$ such that $2 \sigma \max _{k} \mu_{k}^{h}$ $<1$,

$$
E\left(e^{\sigma Z(t, x)}\right)=\left(\operatorname{det}_{2}(I-2 \sigma A(t, x))\right)^{-\frac{1}{2}}<\infty,
$$

where det $_{2}$ denotes the Carleman-Fredholm determinant.

We now introduce a new assumption, as follows:

(H4) $2 \max _{k} \mu_{k}^{h}<1$, for any $h \in K_{y}^{\min }$.

The preceding discussion leads to the next statement.

Proposition 3.6 Assume (H1), (H2) and (H4). For any $h \in K_{y}^{\min }$ there exists $p \in(1, \infty)$ such that,

$$
E\left(\exp \left(p \lambda^{h} S^{0, h}(t, x)\right)\right)<+\infty .
$$

The results of Propositions 3.5, 3.6 and Hölder's inequality yield 
Proposition 3.7 Assume (H1), (H2) and (H4). For any $h \in K_{y}^{\min }$ there exist $p \in(1, \infty)$ and $\rho \in(0,1]$ such that, for any $\beta \in(6, \infty)$,

$$
\sup _{0<\varepsilon \leq 1} E\left(\exp \left(p \lambda^{h} S^{\varepsilon, h}(t, x)\right) \chi_{\rho, \beta}^{h}\left(\varepsilon, \omega+\frac{h}{\varepsilon}\right)\right)<+\infty .
$$

The Taylor development of $p_{t, x}^{\varepsilon, 2}(y)$ relies on the particular integration by parts formula stated in the next Propositions.

Let $\mathbb{E}$ be a Polish space. A family of $\mathbb{E}$-valued random vectors $\left\{U^{i}, i \in I\right\}$ indexed by $I \subset \mathbb{R}$ is said to be uniformly in $\mathbb{D}^{\infty}(\mathbb{E})$ if, for any $p \in(1, \infty)$, $k \geq 1, \sup _{i}\left\|U^{i}\right\|_{p, k} \leq C_{p, k}$. We write $\mathbb{D}^{\infty}$ for $\mathbb{D}^{\infty}(\mathbb{R})$.

For any $n \in \mathbb{N}$, let $\varphi_{n}(x) \in \mathcal{C}_{0}^{\infty}$ be a function satisfying $\left\|\varphi_{n}\right\|_{\infty} \leq 1$,

$$
\varphi_{n}(x)= \begin{cases}1, & \text { if }|x| \leq n \\ 0, & \text { if }|x|>n+1\end{cases}
$$

and $\sup _{n, k \geq 1}\left\|\varphi_{n}^{(k)}\right\|_{\infty} \leq C$.

Let $\left\{G^{\eta}, \eta \in[0,1]\right\},\left\{F^{\zeta}, \zeta \in[0,1]\right\}$ be families of random variables which are uniformly in $\mathbb{D}^{\infty}$. We also assume sup $p_{0 \leq \zeta \leq 1}\left\|,{ }_{F}^{-1}\right\|_{p} \leq C_{p}$, for any $p \in(1, \infty)$. Consider a family $\left\{L^{\varepsilon}, \varepsilon \in(0,1]\right\}$ of random variables. Fix $h \in K_{y}^{\min }$ and $\lambda^{h}$ given in Lemma 4.2 , set $B_{n}=\left\{\lambda^{h} \max \left(\left|S^{\varepsilon, h}(t, x)\right|,\left|S^{0, h}(t, x)\right|\right) \leq n\right\}$. We assume the following properties:

(i) For any $n \geq 1,\left(L^{\varepsilon} \varphi_{n}\left(\lambda^{h} S^{\varepsilon, h}(t, x)\right) \varphi_{n}\left(\lambda^{h} S^{0, h}(t, x)\right)\right) \in \mathbb{D}^{\infty}$.

(ii) For any $\varepsilon \in(0,1]$,

$$
\left|L^{\varepsilon}\right| \leq \exp \left(\lambda^{h} S^{0, h}(t, x)\right) L_{0,1}^{\varepsilon}+\exp \left(\lambda^{h} S^{\varepsilon, h}(t, x)\right) L_{0,2}^{\varepsilon},
$$

where $\left\{L_{0, i}^{\varepsilon}, \varepsilon \in(0,1]\right\}, i=1,2$, are uniformly in $\mathbb{D}^{\infty}$.

(iii) On every set $B_{n}, n \geq 1$,

$$
\left|D^{k} L^{\varepsilon}\right| \leq \exp \left(\lambda^{h} S^{0, h}(t, x)\right) L_{k, 1}^{\varepsilon}+\exp \left(\lambda^{h} S^{\varepsilon, h}(t, x)\right) L_{k, 2}^{\varepsilon}+L_{k, 3}^{\varepsilon},
$$

with $\left\{L_{k, i}^{\varepsilon}, \varepsilon \in(0,1]\right\}, i=1,2,3$, uniformly in $\mathbb{D}^{\infty}\left(L^{2}\left(([0, T] \times[0,1])^{k}\right)\right)$.

Condition (i) above clearly yields $L^{\varepsilon} \in \mathbb{D}_{l o c}^{\infty}$ with localizing sequence $B_{n}$.

Set

$$
\begin{aligned}
& \tilde{\Psi}^{\varepsilon, \eta, \rho}=G^{\eta} L^{\varepsilon} \chi_{\rho, \beta}^{h}\left(\varepsilon, \omega+\frac{h}{\varepsilon}\right), \\
& \Theta_{0}^{\varepsilon, \eta, \zeta, \rho}=\tilde{\Psi}^{\varepsilon, \eta, \rho}, \\
& \Theta_{k}^{\varepsilon, \eta, \zeta, \rho}=\Theta_{k-1}^{\varepsilon, \eta, \zeta, \rho} \delta\left(\left\|D F^{\zeta}\right\|^{-2} D F^{\zeta}\right)-\left\langle D \Theta_{k-1}^{\varepsilon, \eta, \zeta, \rho},\left\|D F^{\zeta}\right\|^{-2} D F^{\zeta}\right\rangle,
\end{aligned}
$$


$\eta, \zeta \in[0,1], \varepsilon \in(0,1], k \geq 1, \beta \in(6, \infty), h \in \mathcal{H}$. Notice that, since $D$ is a local operator $\Theta_{k}^{\varepsilon, \eta, \zeta, \rho}, k \geq 1$, is well defined.

Proposition 3.8 Assume (H1), (H2) and (H4). Let $f \in \mathcal{C}_{b}^{\infty}(\mathbb{R})$. There exists $\rho \in(0,1]$ such that, for any integer $k \geq 1, \eta, \zeta \in[0,1], \varepsilon \in(0,1]$, $h \in K_{y}^{\min }$,

$$
E\left\{f^{(k)}\left(F^{\zeta}\right) G^{\eta} L^{\varepsilon} \chi_{\rho, \beta}^{h}\left(\varepsilon, \omega+\frac{h}{\varepsilon}\right)\right\}=E\left(f\left(F^{\zeta}\right) \Theta_{k}^{\varepsilon, \eta, \zeta, \rho}\right) .
$$

\section{Moreover,}

$$
\sup _{\substack{0 \leq \eta, \zeta \leq 1 \\ 0<\varepsilon \leq 1}}\left\|\Theta_{k}^{\varepsilon, \eta, \zeta, \rho}\right\|_{1}<\infty
$$

Proof: Set

$$
A_{n}=E\left\{f^{(k)}\left(F^{\zeta}\right) G^{\eta} L^{\varepsilon} \chi_{\rho, \beta}^{h}\left(\varepsilon, \omega+\frac{h}{\varepsilon}\right) \varphi_{n}\left(\lambda^{h} S^{\varepsilon, h}(t, x)\right) \varphi_{n}\left(\lambda^{h} S^{0, h}(t, x)\right)\right\} .
$$

Assume that, for any $n \in \mathbb{N}, \zeta, \eta \in[0,1], \varepsilon \in(0,1]$ we can prove the existence of $\rho \in(0,1]$ and a random variable $\Theta_{k, n}^{\varepsilon, \eta, \zeta, \rho} \in L^{1}(\Omega)$ such that $A_{n}=$ $E\left(f\left(F^{\zeta}\right) \Theta_{k, n}^{\varepsilon, \eta, \zeta, \rho}\right), \sup _{\varepsilon, \eta, \zeta, n}\left\|\Theta_{k, n}^{\varepsilon, \eta, \zeta, \rho}\right\|_{1}<\infty$ and $L^{1}-\lim _{n \rightarrow \infty} \Theta_{k, n}^{\varepsilon, \eta, \zeta, \rho}=$ $\Theta_{k}^{\varepsilon, \eta, \zeta, \rho}$ uniformly in $\varepsilon, \eta, \zeta$. Then (3.29) follows.

Indeed, clearly

$$
\lim _{n \rightarrow \infty} E\left(f\left(F^{\zeta}\right) \Theta_{k, n}^{\varepsilon, \eta, \zeta, \rho}\right)=E\left(f\left(F^{\zeta}\right) \Theta_{k}^{\varepsilon, \eta, \zeta, \rho}\right) .
$$

The function $\chi_{\rho, \beta}^{h}\left(\varepsilon, \omega+\frac{h}{\varepsilon}\right)$ satisfies

$$
\begin{aligned}
& 1_{\left\{\left\|X^{\varepsilon, h}-\Psi_{X_{0}}^{h}\right\|_{L^{\beta}([0, T] \times[0,1])}^{\beta} \leq \frac{\rho}{2}\right\}} \leq \chi_{\rho, \beta}^{h}\left(\varepsilon, \omega+\frac{h}{\varepsilon}\right) \\
& \leq 1_{\left\{\left\|X^{\epsilon, h}-\Psi_{X_{0}}^{h}\right\|_{L^{\beta}([0, T] \times[0,1])}^{\beta} \leq \rho\right\}} .
\end{aligned}
$$

Consequently, the random variable $\tilde{\Psi}^{\varepsilon, \eta, \rho}$ belongs to $L^{1}(\Omega)$. Indeed, let $p \in(1, \infty), 2 \rho \in(0,1]$, satisfying the conclusions of Propositions 3.6, 3.7, $1 / q+1 / p=1$. Then, by (3.30), Hölder's inequality and (3.26)

$$
\begin{aligned}
E\left|\tilde{\Psi}^{\varepsilon, \eta, \rho}\right| & \leq\left\|G^{\eta} L_{0,1}^{\varepsilon}\right\|_{q}\left\{E\left(\exp \left(p \lambda^{h} S^{0, h}(t, x)\right)\right)\right\}^{1 / p} \\
& +\left\|G^{\eta} L_{0,2}^{\varepsilon}\right\|_{q}\left\{E\left(\exp \left(p \lambda^{h} S^{\varepsilon, h}(t, x)\right) \chi_{2 \rho, \beta}^{h}\left(\varepsilon, \omega+\frac{h}{\varepsilon}\right)\right)\right\}^{1 / p}<\infty .
\end{aligned}
$$


Thus, by bounded convergence

$$
\lim _{n \rightarrow \infty} A_{n}=E\left\{f^{(k)}\left(F^{\zeta}\right) G^{\eta} L^{\varepsilon} \chi_{\rho, \beta}^{h}\left(\varepsilon, \omega+\frac{h}{\varepsilon}\right)\right\} .
$$

Set $\tilde{\Psi}_{n}^{\varepsilon, \eta, \rho}=G^{\eta} L^{\varepsilon} \chi_{\rho, \beta}^{h}\left(\varepsilon, \omega+\frac{h}{\varepsilon}\right) \varphi_{n}\left(\lambda^{h} S^{\varepsilon, h}(t, x)\right) \varphi_{n}\left(\lambda^{h} S^{0, h}(t, x)\right)$.

Notice that $\tilde{\Psi}_{n}^{\varepsilon, \eta, \rho} \in \mathbb{D}^{\infty}$; then the integration by parts formula yields

$$
A_{n}=E\left(f\left(F^{\zeta}\right) H_{k}\left(F^{\zeta}, \tilde{\Psi}_{n}^{\varepsilon, \eta, \rho}\right)\right),
$$

with $H_{k}\left(F^{\zeta}, \tilde{\Psi}_{n}^{\varepsilon, \eta, \rho}\right)$ defined recurrently as follows:

$$
\begin{aligned}
& H_{1}\left(F^{\zeta}, \tilde{\Psi}_{n}^{\varepsilon, \eta, \rho}\right)=\delta\left(\tilde{\Psi}_{n}^{\varepsilon, \eta, \rho}\left\|D F^{\zeta}\right\|^{-2} D F^{\zeta}\right), \\
& H_{k}\left(F^{\zeta}, \tilde{\Psi}_{n}^{\varepsilon, \eta, \rho}\right)=H_{1}\left(F^{\zeta}, H_{k-1}\left(F^{\zeta}, \tilde{\Psi}_{n}^{\varepsilon, \eta, \rho}\right)\right), \quad k \geq 2 .
\end{aligned}
$$

where $\delta$ is the Skorohod integral.

The properties of the anticipating stochastic calculus imply

$$
\begin{aligned}
H_{1}\left(F^{\zeta}, H_{k-1}\left(F^{\zeta}, \tilde{\Psi}_{n}^{\varepsilon, \eta, \rho}\right)\right)= & H_{k-1}\left(F^{\zeta}, \tilde{\Psi}_{n}^{\varepsilon, \eta, \rho}\right) \delta\left(\left\|D F^{\zeta}\right\|^{-2} D F^{\zeta}\right) \\
& -\left\langle D H_{k-1}\left(F^{\zeta}, \tilde{\Psi}_{n}^{\varepsilon, \eta, \rho}\right),\left\|D F^{\zeta}\right\|^{-2} D F^{\zeta}\right\rangle, \quad k \geq 2 .
\end{aligned}
$$

Define

$$
\begin{aligned}
\Theta_{0, n}^{\varepsilon, \eta, \zeta, \rho} & =\tilde{\Psi}_{n}^{\varepsilon, \eta, \rho}, \\
\Theta_{k, n}^{\varepsilon, \eta, \zeta, \rho} & =\Theta_{k-1, n}^{\varepsilon, \eta, \zeta, \rho} \delta\left(\left\|D F^{\zeta}\right\|^{-2} D F^{\zeta}\right)-\left\langle D \Theta_{k-1, n}^{\varepsilon, \eta, \zeta, \rho},\left\|D F^{\zeta}\right\|^{-2} D F^{\zeta}\right\rangle,
\end{aligned}
$$

for any $k \geq 1$.

Clearly, by the local property of the derivative operator $D$,

$$
\Theta_{k, n}^{\varepsilon, \eta, \zeta, \rho}=\Theta_{k}^{\varepsilon, \eta, \zeta, \rho}, \quad \text { on } B_{n}, \quad \text { for any } k \geq 0 .
$$

We want to prove the following facts. There exists $\rho \in(0,1]$ and a decreasing sequence $p_{k} \in(1, \infty), k \geq 0$, such that

$$
\begin{array}{ll}
\sup _{\varepsilon, \eta, \zeta} & \left\|\Theta_{k, n}^{\varepsilon, \eta, \zeta, \rho}-\Theta_{k}^{\varepsilon, \eta, \zeta, \rho}\right\|_{p_{k}} \underset{n \rightarrow \infty}{\longrightarrow} 0, \\
\sup _{\varepsilon, \eta, \zeta, n} & \left\|\Theta_{k, n}^{\varepsilon, \eta, \zeta, \rho}\right\|_{p_{k}} \leq C .
\end{array}
$$


This clearly suffices to end the proof.

We check (3.31), (3.32) using induction on $k$. Let $k=0$, then

$$
T_{0}^{\varepsilon, \eta, n}:=\left\|\tilde{\Psi}_{n}^{\varepsilon, \eta, \rho}-\tilde{\Psi}^{\varepsilon, \eta, \rho}\right\|_{p_{0}}=\left\|\left(\tilde{\Psi}_{n}^{\varepsilon, \eta, \rho}-\tilde{\Psi}^{\varepsilon, \eta, \rho}\right) 1_{B_{n}^{c}}\right\|_{p_{0}} .
$$

Fix $p>1$ and $\rho \in(0,1]$ satisfying Propositions 3.6, 3.7. Let $p_{0}, q_{1}, q_{2}, q_{3}>1$ such that $1 / q_{1}+1 / q_{2}+1 / q_{3}=1, p_{0} q_{2}=p$. Then, Hölder's inequality, (3.30), Propositions 3.6, 3.7 and (3.26) yield

$$
\begin{aligned}
T_{0}^{\varepsilon, \eta, n} \leq & \left(\left\|G^{\eta} L_{0,1}^{\varepsilon}\right\|_{p_{0} q_{1}}\left\{E\left(\exp \left(\lambda^{h} p_{0} q_{2} S^{0, h}(t, x)\right)\right)\right\}^{\frac{1}{p_{0} q_{2}}}\right. \\
& \left.+\left\|G^{\eta} L_{0,2}^{\varepsilon}\right\|_{p_{0} q_{1}}\left\{E\left(\exp \left(\lambda^{h} p_{0} q_{2} S^{\varepsilon, h}(t, x)\right) \chi_{2 \rho, \beta}^{h}\left(\varepsilon, \omega+\frac{h}{\varepsilon}\right)\right)\right\}^{\frac{1}{p_{0} q_{2}}}\right) \\
& \times\left(P\left(B_{n}^{c}\right)\right)^{\frac{1}{p_{0} q_{3}}} .
\end{aligned}
$$

Thus

$$
\sup _{\varepsilon, \eta} T_{0}^{\varepsilon, \eta, n} \underset{n \rightarrow \infty}{\longrightarrow} 0
$$

The uniform estimate (3.32) follows from (3.26), Hölder's inequality, (3.30), Propositions 3.6, 3.7 and the boundness of $\varphi_{n}$ and its derivatives.

In order to simplify the presentation we will give the precise arguments allowing to check (3.31), (3.32) for $k=1$ and only sketch the general case. We have

$$
\left\|\Theta_{1, n}^{\varepsilon, \eta, \zeta, \rho}-\Theta_{1}^{\varepsilon, \eta, \zeta, \rho}\right\|_{p_{1}}^{p_{1}}=\left\|\left(\Theta_{1, n}^{\varepsilon, \eta, \zeta, \rho}-\Theta_{1}^{\varepsilon, \eta, \zeta, \rho}\right) 1_{B_{n}^{c}}\right\|_{p_{1}}^{p_{1}} \leq \sum_{i=1}^{6} T_{i}^{\varepsilon, \eta, \zeta, n}
$$


where

$$
\begin{aligned}
& T_{1}^{\varepsilon, \eta, \zeta, n}= E\left|\left(\tilde{\Psi}_{n}^{\varepsilon, \eta, \rho}-\tilde{\Psi}^{\varepsilon, \eta, \rho}\right) \delta\left(\left\|D F^{\zeta}\right\|^{-2} D F^{\zeta}\right)\right|^{p_{1}}, \\
& T_{2}^{\varepsilon, \eta, \zeta, n}=E \mid L^{\varepsilon} \chi_{\rho, \beta}^{h}\left(\varepsilon, \omega+\frac{h}{\varepsilon}\right)\left\langle D G^{\eta},\left\|D F^{\zeta}\right\|^{-2} D F^{\zeta}\right\rangle\left(1-\varphi_{n}\left(\lambda^{h} S^{\varepsilon, h}(t, x)\right)\right.\left.\quad \quad \varphi_{n}\left(\lambda^{h} S^{0, h}(t, x)\right)\right)\left.\right|^{p_{1}}, \\
& T_{3}^{\varepsilon, \eta, \zeta, n}=E \mid G^{\eta} \chi_{\rho, \beta}^{h}\left(\varepsilon, \omega+\frac{h}{\varepsilon}\right)\left\langle D L^{\varepsilon},\left\|D F^{\zeta}\right\|^{-2} D F^{\zeta}\right\rangle\left(1-\varphi_{n}\left(\lambda^{h} S^{\varepsilon, h}(t, x)\right)\right.\left.\quad \quad \varphi_{n}\left(\lambda^{h} S^{0, h}(t, x)\right)\right)\left.\right|^{p_{1}}, \\
& T_{4}^{\varepsilon, \eta, \zeta, n}=E \mid G^{\eta} L^{\varepsilon}\left\langle D \chi_{\rho, \beta}^{h}\left(\varepsilon, \omega+\frac{h}{\varepsilon}\right),\left\|D F^{\zeta}\right\|^{-2} D F^{\zeta}\right\rangle\left(1-\varphi_{n}\left(\lambda^{h} S^{\varepsilon, h}(t, x)\right)\right. \\
&\left.\quad \times \quad \varphi_{n}\left(\lambda^{h} S^{0, h}(t, x)\right)\right)\left.\right|^{p_{1}}, \\
& T_{5}^{\varepsilon, \eta, \zeta, n}=E \mid G^{\eta} L^{\varepsilon} \chi_{\rho, \beta}^{h}\left(\varepsilon, \omega+\frac{h}{\varepsilon}\right) \varphi_{n}^{\prime}\left(\lambda^{h} S^{\varepsilon, h}(t, x)\right) \varphi_{n}\left(\lambda^{h} S^{0, h}(t, x)\right) \\
& \quad \times\left\langle\lambda^{h} D S^{\varepsilon, h}(t, x),\left\|D F^{\zeta}\right\|^{-2} D F^{\zeta}\right\rangle \times\left. 1_{B_{n}^{c}}\right|^{p_{1}}, \\
& T_{6}^{\varepsilon, \eta, \zeta, n}=E \mid G^{\eta} L^{\varepsilon} \chi_{\rho, \beta}^{h}\left(\varepsilon, \omega+\frac{h}{\varepsilon}\right) \varphi_{n}\left(\lambda^{h} S^{\varepsilon, h}(t, x)\right) \varphi_{n}^{\prime}\left(\lambda^{h} S^{0, h}(t, x)\right) \\
& \quad \times\left\langle\lambda^{h} D S^{0, h}(t, x),\left\|D F^{\zeta}\right\|^{-2} D F^{\zeta}\right\rangle \times\left. 1_{B_{n}^{c}}\right|^{p_{1}} .
\end{aligned}
$$

Choose $p_{1}, q>1$ with $p_{1} q=p_{0}$. Then, Hölder's inequality yields

$$
\sup _{\varepsilon, \eta, \zeta} T_{1}^{\varepsilon, \eta, \zeta, n} \leq C \sup _{\varepsilon, \eta, \zeta}\left\|\tilde{\Psi}_{n}^{\varepsilon, \eta, \rho}-\tilde{\Psi}^{\varepsilon, \eta, \rho}\right\|_{p_{0}}^{p_{1}} \underset{n \rightarrow \infty}{\longrightarrow} 0 .
$$

Fix $p>1$ and $2 \rho \in(0,1]$ satisfying Propositions $3.6,3.7$. Let $p_{1}, q_{1}, q_{2}, q_{3}>1$ such that $1 / q_{1}+1 / q_{2}+1 / q_{3}=1, p_{1} q_{1}=p$. Then, (3.26), (3.27), Hölder's inequality, (3.30) and Propositions 3.6, 3.7 imply

$$
\sup _{\varepsilon, \eta, \zeta} T_{i}^{\varepsilon, \eta, \zeta, n} \leq C\left(P\left(B_{n}^{c}\right)\right)^{\frac{1}{q_{3}}}, \quad i=2, \cdots, 6 .
$$

Thus (3.31) holds for $k=1$ and (3.32) follows from (3.26), (3.27), Hölder's inequality, (3.30), Propositions 3.6, 3.7 and the boundedness of $\varphi_{n}$ and its derivatives.

For a general $k$ we only give some remarks. First, from the definitions of $\Theta_{k, n}^{\varepsilon, \eta, \zeta, \rho}, \Theta_{k}^{\varepsilon, \eta, \zeta, \rho}$, we have

$$
\left\|\Theta_{k, n}^{\varepsilon, \eta, \zeta, \rho}-\Theta_{k}^{\varepsilon, \eta, \zeta, \rho}\right\|_{p_{k}}^{p_{k}} \leq A_{1}^{\varepsilon, \eta, \zeta, n}+A_{2}^{\varepsilon, \eta, \zeta, n},
$$


with

$$
\begin{aligned}
& A_{1}^{\varepsilon, \eta, \zeta, n}=E\left|\left(\Theta_{k-1, n}^{\varepsilon, \eta, \zeta, \rho}-\Theta_{k-1}^{\varepsilon, \eta, \zeta, \rho}\right) \delta\left(\left\|D F^{\zeta}\right\|^{-2} D F^{\zeta}\right)\right|^{p_{k}} \\
& A_{2}^{\varepsilon, \eta, \zeta, n}=E\left|\left\langle D\left(\Theta_{k-1, n}^{\varepsilon, \eta, \zeta, \rho}-\Theta_{k-1}^{\varepsilon, \eta, \zeta, \rho}\right),\left\|D F^{\zeta}\right\|^{-2} D F^{\zeta}\right\rangle 1_{B_{n}^{c}}\right|^{p_{k}}
\end{aligned}
$$

Hölder's inequality and the inductive assumption yield $\sup _{\varepsilon, \eta, \zeta} A_{1}^{\varepsilon, \eta, \zeta, n} \longrightarrow 0$ as $n \rightarrow \infty$. To study $A_{2}^{\varepsilon, \eta, \zeta, n}$ we note

$$
\begin{aligned}
& \left|\left\langle D\left(\Theta_{k-1, n}^{\varepsilon, \eta, \zeta, \rho}-\Theta_{k-1}^{\varepsilon, \eta, \zeta, \rho}\right),\left\|D F^{\zeta}\right\|^{-2} D F^{\zeta}\right\rangle\right| \\
& \quad \leq \exp \left(\lambda^{h} S^{\varepsilon, h}(t, x)\right) \chi_{2 \rho, \beta}^{h}\left(\varepsilon, \omega+\frac{h}{\varepsilon}\right) \Phi_{1}^{\varepsilon, \eta, \zeta}+\exp \left(\lambda^{h} S^{0, h}(t, x)\right) \Phi_{2}^{\varepsilon, \eta, \zeta},
\end{aligned}
$$

with $\Phi_{1}^{\varepsilon, \eta, \zeta}, \Phi_{2}^{\varepsilon, \eta, \zeta}$ uniformly in $\mathbb{D}^{\infty}$. Therefore, since $\lim _{n \rightarrow \infty} P\left(B_{n}^{c}\right)=0$, Hölder's inequality and Propositions 3.6, 3.7 show that

$$
\lim _{n \rightarrow \infty}\left(\sup _{\varepsilon, \eta, \zeta} A_{2}^{\varepsilon, \eta, \zeta, n}\right)=0 \text {. }
$$

This ends the proof.

With less effort, using similar arguments as in the previous Proposition, we can prove the next Proposition 3.9. Let $F^{\zeta}, G^{\eta}$ be as in Proposition 3.8.

Set

$$
\begin{aligned}
& \tilde{\Phi}^{\eta}=G^{\eta} \exp \left(\lambda^{h} S^{0, h}(t, x)\right), \\
& \Theta_{0}^{\eta, \zeta}=\tilde{\Phi}^{\eta}, \\
& \Theta_{k}^{\eta, \zeta}=\Theta_{k-1}^{\eta, \zeta} \delta\left(\left\|D F^{\zeta}\right\|^{-2} D F^{\zeta}\right)-\left\langle D \Theta_{k-1}^{\eta, \zeta},\left\|D F^{\zeta}\right\|^{-2} D F^{\zeta}\right\rangle,
\end{aligned}
$$

$\eta, \zeta \in[0,1], k \geq 1, h \in \mathcal{H}$.

Proposition 3.9 Assume $(\mathrm{H} 1),(\mathrm{H} 2)$ and $(\mathrm{H} 4)$. Let $f \in \mathcal{C}_{b}^{\infty}(\mathbb{R})$. For every integer $k \geq 1$ and $\eta, \zeta \in[0,1]$,

$$
E\left\{f^{(k)}\left(F^{\zeta}\right) G^{\eta} \exp \left(\lambda^{h} S^{0, h}(t, x)\right)\right\}=E\left(f\left(F^{\zeta}\right) \Theta_{k}^{\eta, \zeta}\right)
$$

and $\sup _{0 \leq \eta, \zeta \leq 1}\left\|\Theta_{k}^{\eta, \zeta}\right\|_{1}<\infty$.

Let $f: \mathbb{R} \rightarrow \mathbb{R}$ be a $\mathcal{C}^{\infty}$ function and assume $\varepsilon \rightarrow F^{\varepsilon}$ is a $\mathcal{C}^{\infty}((0,1) ; \mathbb{R})$ mapping. Leibniz's formula yields, for any integer $j \geq 1$,

$$
\frac{d^{j}}{d \varepsilon^{j}}\left(f\left(F^{\varepsilon}\right)\right)=\sum^{(j)} f^{\left(k_{j}\right)}\left(F^{\varepsilon}\right) \prod_{l=1}^{k_{j}} F^{\varepsilon, \beta_{l}},
$$


where the symbol $\sum^{(j)}$ is a shorthand for

$$
\sum_{k_{j}=1}^{j} \sum_{\substack{\beta_{1}+\ldots+\beta_{k_{j}}=j \\ \beta_{1}, \ldots, \beta_{k_{j}} \geq 1}} c_{j}\left(\beta_{1}, \ldots, \beta_{k_{j}}\right)
$$

and $F^{\varepsilon, j}=d^{j} F^{\varepsilon} / d \varepsilon^{j}$. The coefficients $c_{j}\left(\beta_{1}, \ldots, \beta_{k_{j}}\right)$ are computed by induction.

Let $\delta \in(0, \rho), \rho$ as in Proposition 3.7, and $\beta \in(6, \infty)$. Define

$$
p_{t, x, \bar{h}}^{\varepsilon, 2}(0)=E\left\{\exp \left(\bar{\lambda} S^{\varepsilon, \bar{h}}(t, x)\right) \chi_{\delta, \beta}^{\bar{h}}\left(\varepsilon, \omega+\frac{\bar{h}}{\varepsilon}\right) \delta_{\{0\}}\left(\hat{X}^{\varepsilon, \bar{h}}(t, x)\right)\right\} .
$$

Notice that, by (3.6), $p_{t, x}^{\varepsilon, 2}(y)=\varepsilon^{-1} \exp \left\{-\|\bar{h}\|_{\mathcal{H}}^{2} /\left(2 \varepsilon^{2}\right)\right\} p_{t, x, \bar{h}}^{\varepsilon, 2}(0)$.

Let $\phi$ be a nondegenerate random variable and $\psi \in \mathbb{D}_{l o c}^{\infty}$. We set

$$
\begin{aligned}
\tilde{H}_{0}(\phi, \psi)= & \psi \\
\tilde{H}_{k}(\phi, \psi)= & \tilde{H}_{k-1}(\phi, \psi) \delta\left(D \phi\|D \phi\|^{-2}\right) \\
& -\left\langle D \tilde{H}_{k-1}(\phi, \psi), D \phi\|D \phi\|^{-2}\right\rangle,
\end{aligned}
$$

$k \geq 1$.

We remark that the sequences $\Theta_{k}^{\varepsilon, \eta, \zeta, \rho}, \Theta_{k}^{\eta, \zeta}$, defined in (3.28) and (3.33), respectively, can be described in terms of the operator $\tilde{H}_{k}$.

Proposition 3.10 Assume (H1)-(H4). Then, we have

$$
p_{t, x, \bar{h}}^{\varepsilon, 2}(0)=p_{t, x, \bar{h}}^{0}+\sum_{i=1}^{n} \varepsilon^{i} p_{t, x, \bar{h}}^{i}+\varepsilon^{n+1} \tilde{p}_{t, x, \bar{h}}^{n+1, \varepsilon},
$$

$n \geq 0$.

The coefficients $p_{t, x, \bar{h}}^{i}$ are null for odd $i$. For any even $i \in\{0, \cdots, n\}$,

$$
\begin{aligned}
p_{t, x, \bar{h}}^{0}= & E\left\{1_{\left\{X_{1}^{0, \bar{h}}(t, x)>0\right\}} \tilde{H}_{1}\left(X_{1}^{0, \bar{h}}(t, x), \exp \left(\bar{\lambda} S^{0, \bar{h}}(t, x)\right)\right)\right\} \\
p_{t, x, \bar{h}}^{i}= & \sum_{j=1}^{i} \frac{1}{j !(i-j) !} \sum \sum^{(j)} \sum^{(i-j)} \bar{\lambda}^{k_{i-j}} E\left\{1 _ { \{ X _ { 1 } ^ { 0 , \overline { h } } ( t , x ) > 0 \} } \tilde { H } _ { k _ { j } + 1 } \left(X_{1}^{0, \bar{h}}(t, x),\right.\right. \\
& \left.\left.\quad \exp \left(\bar{\lambda} S^{0, \bar{h}}(t, x)\right) \prod_{l=1}^{k_{j}} \frac{X_{\beta_{l}+1}^{0, \bar{h}}(t, x)}{\beta_{l}+1} \prod_{l^{\prime}=1}^{k_{i-j}} \frac{X_{\beta_{l^{\prime}+2}+\bar{h}}(t, x)}{\left(\beta_{l^{\prime}}+1\right)\left(\beta_{l^{\prime}}+2\right)}\right)\right\}
\end{aligned}
$$




\section{Furthermore,}

$$
\sup _{0<\varepsilon \leq 1}\left|\tilde{p}_{t, x, \bar{h}}^{n+1, \varepsilon}\right|<C .
$$

Proof : Let $f$ be a $\mathcal{C}^{\infty}$ function with compact support and symmetric. Set

$$
M_{t, x}^{\varepsilon}=E\left\{\exp \left(\bar{\lambda} S^{\varepsilon, \bar{h}}(t, x)\right) \chi_{\delta, \beta}^{\bar{h}}\left(\varepsilon, \omega+\frac{\bar{h}}{\varepsilon}\right) f\left(\hat{X}^{\varepsilon, \bar{h}}(t, x)\right)\right\} .
$$

The chain rule (3.34) and (2.11) yield the Taylor expansion

$$
\begin{aligned}
f\left(\hat{X}^{\varepsilon, \bar{h}}(t, x)\right) & =f\left(X_{1}^{0, \bar{h}}(t, x)\right)+\sum_{j=1}^{N} \varepsilon^{j} \frac{1}{j !} \sum^{(j)} f^{\left(k_{j}\right)}\left(X_{1}^{0, \bar{h}}(t, x)\right) \\
& \times \prod_{l=1}^{k_{j}} \frac{1}{\beta_{l}+1} X_{\beta_{l}+1}^{0, \bar{h}}(t, x)+\varepsilon^{N+1} \int_{0}^{1} \frac{(1-\xi)^{N}}{N !} \\
& \times \sum^{(N+1)} f^{\left(k_{N+1}\right)}\left(\hat{X}^{\varepsilon \xi, \bar{h}}(t, x)\right) \prod_{l=1}^{k_{N+1}} \hat{X}_{\beta_{l}, \xi, \bar{h}}(t, x) d \xi .
\end{aligned}
$$

Analogously, for each $\delta$ and $\beta$,

$$
\chi_{\delta, \beta}^{\bar{h}}\left(\varepsilon, \omega+\frac{\bar{h}}{\varepsilon}\right)=1+\varepsilon^{N+1} R_{N+1}^{1, \varepsilon}(t, x),
$$

where $\left\{R_{N+1}^{1, \varepsilon}(t, x), \varepsilon \in(0,1]\right\}$ uniformly in $\mathbb{D}^{\infty}$, as can be easily checked.

In order to study the term $\exp \left(\bar{\lambda} S^{\varepsilon, \bar{h}}(t, x)\right)$ we use the Taylor expansion $e^{x}=\sum_{j=0}^{N} x^{j} / j !+r_{N+1}(x)$ with $\left|r_{N+1}(x)\right| \leq\left(e^{x}+1\right)|x|^{N+1} /(N+1)$ !, for $x=\bar{\lambda}\left(S^{\varepsilon, \bar{h}}-S^{0, \bar{h}}\right)(t, x)$. Then, since $S^{\varepsilon, \bar{h}}(t, x)$ is $\mathcal{C}^{\infty}$ with respect to $\varepsilon$, using (2.12), (2.16), we obtain

$$
\begin{aligned}
\exp \left(\bar{\lambda} S^{\varepsilon, \bar{h}}(t, x)\right) & =\exp \left(\bar{\lambda} S^{0, \bar{h}}(t, x)\right)\left\{\sum_{j=0}^{N} \frac{\varepsilon^{j}}{j !} G_{j}+\varepsilon^{N+1} G_{N+1}^{\varepsilon}\right\} \\
& +\varepsilon^{N+1} R_{N+1}^{2, \varepsilon}(t, x)
\end{aligned}
$$

where $G_{j}=\sum^{(j)} \bar{\lambda}^{k_{j}} \prod_{l=1}^{k_{j}} \frac{1}{\left(\beta_{l}+1\right)\left(\beta_{l}+2\right)} X_{\beta_{l}+2}^{0, \bar{h}}(t, x) \in \mathbb{D}^{\infty},\left\{G_{N+1}^{\varepsilon}, \varepsilon \in\right.$ $(0,1]\}$ is uniformly in $\mathbb{D}^{\infty}$ and

$$
\begin{aligned}
& \left|R_{N+1}^{2, \varepsilon}(t, x)\right| \\
& \quad \leq \exp \left(\lambda S^{0, h}(t, x)\right) R_{N+1,1}^{2, \varepsilon, 0}(t, x)+\exp \left(\lambda S^{\varepsilon, h}(t, x)\right) R_{N+1,2}^{2, \varepsilon, 0}(t, x),
\end{aligned}
$$


with $\left\{R_{N+1, i}^{2, \varepsilon, 0}(t, x), \varepsilon \in(0,1]\right\}, i=1,2$, uniformly in $\mathbb{D}^{\infty}$.

From (3.39) it follows that $R_{N+1}^{2, \varepsilon}(t, x) \varphi_{n}\left(\bar{\lambda} S^{\varepsilon, \bar{h}}(t, x)\right) \varphi_{n}\left(\bar{\lambda} S^{0, \bar{h}}(t, x)\right)$, with $\varphi_{n}$ defined (3.25), belongs to $\mathbb{D}^{\infty}$. Moreover, on $B_{n}$,

$$
\begin{aligned}
\left|D^{k} R_{N+1}^{2, \varepsilon}(t, x)\right| \leq & \exp \left(\lambda S^{0, h}(t, x)\right) R_{N+1,1}^{2, \varepsilon, k}(t, x)+\exp \left(\lambda S^{\varepsilon, h}(t, x)\right) \\
& \times R_{N+1,2}^{2, \varepsilon, k}(t, x)+R_{N+1,3}^{2, \varepsilon, k}(t, x),
\end{aligned}
$$

with $\left\{R_{N+1, i}^{2, \varepsilon, k}(t, x), \varepsilon \in(0,1]\right\}$ uniformly in $\mathbb{D}^{\infty}\left(L^{2}\left(([0, T] \times[0,1])^{k}\right)\right)$ for any $i=1,2,3, k \geq 1$. This inequality can be easily checked using induction on $k$.

Putting together the expansions (3.37)-(3.39) yields

$$
\begin{aligned}
M_{t, x}^{\varepsilon} & =E\left\{f\left(X_{1}^{0, \bar{h}}(t, x)\right) \exp \left(\bar{\lambda} S^{0, \bar{h}}(t, x)\right)\right\} \\
& +\sum_{i=1}^{n} \varepsilon^{i} \sum_{j=0}^{i} \frac{1}{j !(i-j) !} E\left\{\sum^{(j)} \sum^{(i-j)} f^{\left(k_{j}\right)}\left(X_{1}^{0, \bar{h}}(t, x)\right) \exp \left(\bar{\lambda} S^{0, \bar{h}}(t, x)\right)\right. \\
& \left.\times \bar{\lambda}^{k_{i-j}} \prod_{l=1}^{k_{j}} \frac{X_{\beta_{l}+1}^{0, \bar{h}}(t, x)}{\beta_{l}+1} \prod_{l^{\prime}=1}^{k_{i-j}} \frac{X_{\beta_{l^{\prime}}+2}^{0, \bar{h}}(t, x)}{\left(\beta_{l^{\prime}}+1\right)\left(\beta_{l^{\prime}}+2\right)}\right\} \\
& +\varepsilon^{n+1} R_{n+1}^{3, \varepsilon}(t, x) .
\end{aligned}
$$

Let $\left(f_{n}\right)_{n>1}$ be a sequence of functions satisfying the same requirements as $f$ and converging to $\delta_{\{0\}}$ as $n \rightarrow \infty$. Proposition 3.9 applied to $F^{0}=$ $X_{1}^{0, \bar{h}}(t, x), G^{0}=\bar{\lambda}^{k_{i-j}} \prod_{l=1}^{k_{j}} \frac{X_{\beta_{l}+1}^{0, \bar{h}}(t, x)}{\beta_{l}+1} \prod_{l^{\prime}=1}^{k_{i-j}} \frac{X_{\beta_{l^{\prime}+2}}^{0, \bar{h}}(t, x)}{\left(\beta_{l^{\prime}}+1\right)\left(\beta_{l^{\prime}}+2\right)}$ ensures (3.36). Since the Wiener sheet $W$ has a symmetric law, the odd terms in (3.35) are zero.

As for the rest, a detailed analysis of $R_{n+1}^{3, \varepsilon}(t, x)$ shows that it can be de- 
scribed by means of a finite sum of terms of the following type:

$$
\begin{aligned}
& R_{1}^{\varepsilon}=f^{\left(a_{1}\right)}\left(X_{1}^{0, \bar{h}}(t, x)\right) \exp \left(\bar{\lambda} S^{0, \bar{h}}(t, x)\right) G_{1}^{\varepsilon}, \\
& R_{2}^{\varepsilon}=f^{\left(a_{2}\right)}\left(X_{1}^{0, \bar{h}}(t, x)\right) G_{2}^{\varepsilon} R_{N_{1}}^{2, \varepsilon}(t, x) \chi_{\delta, \beta}^{\bar{h}}\left(\varepsilon, \omega+\frac{\bar{h}}{\varepsilon}\right), \\
& R_{3}^{\varepsilon}=\int_{[0,1]} f^{\left(a_{3}\right)}\left(\hat{X}^{\varepsilon \xi, \bar{h}}(t, x)\right) G_{3}^{\varepsilon \xi} G_{4}^{\varepsilon} \exp \left(\bar{\lambda} S^{0, \bar{h}}(t, x)\right) d \xi \\
& R_{4}^{\varepsilon}=\int_{[0,1]} f^{\left(a_{4}\right)}\left(\hat{X}^{\varepsilon \xi, \bar{h}}(t, x)\right) G_{5}^{\varepsilon \xi} R_{N_{2}}^{2, \varepsilon}(t, x) \chi_{\delta, \beta}^{\bar{h}}\left(\varepsilon, \omega+\frac{\bar{h}}{\varepsilon}\right) d \xi,
\end{aligned}
$$

with $\left\{G_{i}^{\varepsilon}, \varepsilon \in(0,1]\right\}, i=1, \cdots, 5$, uniformly in $\mathbb{D}^{\infty}$ and some positive integers $a_{i}, i=1, \cdots, 4, N_{1}, N_{2}$. Proposition 3.9 ensures sup $\operatorname{poc\varepsilon <1}_{0<}\left|E\left(R_{1}^{\varepsilon}+R_{3}^{\varepsilon}\right)\right| \leq$ $C$, while Proposition 3.8 yields sup $\operatorname{suc\varepsilon }_{0<1}\left|E\left(R_{2}^{\varepsilon}+R_{4}^{\varepsilon}\right)\right| \leq \bar{C}$. This completes the proof.

As a Corollary of Propositions 3.1 and 3.10, we are now ready to state the main result. Let us recall that in (H3), $K_{y}^{\min }=\left\{h_{1}, \cdots, h_{n_{0}}\right\}$ and $a=\left\|h_{j}\right\|_{\mathcal{H}}^{2}$, $j=1, \cdots, n_{0}$.

Theorem 3.11 Assume (H1)-(H4). For any $y \in \mathbb{R}, y \neq \Psi_{X_{0}}^{0}(t, x)$, as $\varepsilon \downarrow 0$,

$$
p_{t, x}^{\varepsilon}(y) \sim \frac{1}{\varepsilon} \exp \left(-\frac{1}{2 \varepsilon^{2}} a\right)\left(p_{t, x}^{0}+\varepsilon p_{t, x}^{1}+\varepsilon^{2} p_{t, x}^{2}+\cdots\right),
$$

where $p_{t, x}^{i}$ are null for odd $i$ and for even $i$

$$
p_{t, x}^{i}=\sum_{j=1}^{n_{0}} p_{t, x, h_{j}}^{i},
$$

where $p_{t, x, h_{j}}^{i}$ are defined in (3.36). The symbol $\sim$ in (3.40) means

$$
\limsup _{\varepsilon \downarrow 0} \frac{p_{t, x}^{\varepsilon}(y)-\frac{1}{\varepsilon} \exp \left(-\frac{1}{2 \varepsilon^{2}} a\right)\left(p_{t, x}^{0}+\varepsilon p_{t, x}^{1}+\cdots+\varepsilon^{k-1} p_{t, x}^{k-1}\right)}{\varepsilon^{k-1} \exp \left(-\frac{1}{2 \varepsilon^{2}} a\right)}<\infty
$$

for any $k \geq 1$.

Remark 3.12. The validity of $(\mathrm{H} 4)$ can be ensured under assumptions involving only the coefficients $\sigma, b$ and the initial value $X_{0}$. Indeed, assume for simplicity that $K_{y}^{\min }=\bar{h}$. Then, condition

$$
\|A(t, x)\|_{H S}^{2}:=\int_{0}^{t} \int_{0}^{1} \int_{0}^{t} \int_{0}^{1} f_{2}^{\bar{h}}\left((t, x) ;\left(r_{1}, z_{1}\right),\left(r_{2}, z_{2}\right)\right)^{2} d r_{1} d z_{1} d r_{2} d z_{2}<\frac{1}{4}
$$


yields (H4), where we have used the notation introduced before Proposition 3.6 and $\|\cdot\|_{H S}$ is the Hilbert-Schmidt norm.

From (3.24) and Lemma 4.2, it follows that

$$
\left\{\begin{array}{l}
f_{2}^{\bar{h}}((t, x) ; \cdot)=\frac{1}{2} \lambda^{\bar{h}} D^{2} S^{0, \bar{h}}(t, x), \\
\|\bar{h}\|_{\mathcal{H}}=\lambda^{\bar{h}}\left\{E\left|X_{1}^{0, \bar{h}}(t, x)\right|^{2}\right\}^{1 / 2} .
\end{array}\right.
$$

The process $\left\{D_{\left(r_{2}, z_{2}\right)\left(r_{1}, z_{1}\right)}^{2} S^{0, \bar{h}}(t, x),\left(\left(r_{2}, z_{2}\right),\left(r_{1}, z_{1}\right)\right) \in([0, T] \times[0,1])^{2}\right\}$ is deterministic and satisfies

$$
\begin{aligned}
& D_{\left(r_{2}, z_{2}\right)\left(r_{1}, z_{1}\right)}^{2} S^{0, \bar{h}}(t, x)=G_{t-r_{1}}\left(x, z_{1}\right) \sigma^{\prime}\left(\Psi_{X_{0}}^{\bar{h}}\left(r_{1}, z_{1}\right)\right) D_{r_{2}, z_{2}} X_{1}^{0, \bar{h}}\left(r_{1}, z_{1}\right) \\
& +G_{t-r_{2}}\left(x, z_{2}\right) \sigma^{\prime}\left(\Psi_{X_{0}}^{\bar{h}}\left(r_{2}, z_{2}\right)\right) D_{r_{1}, z_{1}} X_{1}^{0, \bar{h}}\left(r_{2}, z_{2}\right) \\
& +\int_{r_{1} \vee r_{2}}^{t} \int_{0}^{1} G_{t-s}(x, y) \sigma^{\prime}\left(\Psi_{X_{0}}^{\bar{h}}(s, y)\right) D_{\left(r_{2}, z_{2}\right)\left(r_{1}, z_{1}\right)}^{2} S^{0, \bar{h}}(s, y) \dot{\bar{h}}(s, y) d s d y \\
& +\int_{r_{1} \vee r_{2}}^{t} \int_{0}^{1} G_{t-s}(x, y) \sigma^{\prime \prime}\left(\Psi_{X_{0}}^{\bar{h}}(s, y)\right) D_{r_{2}, z_{2}} X_{1}^{0, \bar{h}}(s, y) \\
& +\int_{r_{1} \vee r_{2}}^{t} \int_{0}^{t} G_{r_{1}, z_{1}} X_{1}^{0, \bar{h}}(s, y) \dot{\bar{h}}(s, y) d s d y \\
& +\int_{r_{1} \vee r_{2}}^{t} \int_{0}^{1} G_{t-s}(x, y) b^{\prime}\left(\Psi_{X_{0}}^{\bar{h}}(s, y)\right) D_{\left(r_{2}, z_{2}\right)\left(r_{1}, z_{1}\right)}^{2} S^{0, \bar{h}}(s, y) d s d y
\end{aligned}
$$

Moreover, (2.2) and the rules of Malliavin Calculus yield

$$
\begin{aligned}
D_{r_{1}, z_{1}} & X_{1}^{0, \bar{h}}(t, x)=G_{t-r_{1}}\left(x, z_{1}\right) \sigma\left(\Psi_{X_{0}}^{\bar{h}}\left(r_{1}, z_{1}\right)\right) \\
& +\int_{r_{1}}^{t} \int_{0}^{1} G_{t-s}(x, y)\left\{\sigma^{\prime}\left(\Psi_{X_{0}}^{\bar{h}}(s, y)\right) D_{r_{1}, z_{1}} X_{1}^{0, \bar{h}}(s, y) \dot{\bar{h}}(s, y)\right. \\
& \left.+b^{\prime}\left(\Psi_{X_{0}}^{\bar{h}_{0}}(s, y)\right) D_{r_{1}, z_{1}} X_{1}^{0, \bar{h}}(s, y)\right\} d s d y .
\end{aligned}
$$

Thus, (3.9) and the standard method based on Hölder's inequality and Gronwall's lemma imply

$$
\left.\left.\sup _{t, x}\left|\int_{0}^{t} \int_{0}^{1}\right| D_{r_{1}, z_{1}} X_{1}^{0, \bar{h}}(t, x)\right|^{2} d r_{1} d z_{1}\right|^{p} \leq C \exp \left\{C\left(\left\|\sigma^{\prime}\right\|_{\infty}^{2 p}+\left\|b^{\prime}\right\|_{\infty}^{2 p}\right)\right\} .
$$


Therefore,

$$
\begin{aligned}
& \left.\left.\left|\int_{0}^{t} \int_{0}^{1} \int_{0}^{t} \int_{0}^{1}\right| D_{\left(r_{2}, z_{2}\right)\left(r_{1}, z_{1}\right)}^{2} S^{0, \bar{h}}(t, x)\right|^{2} d r_{1} d z_{1} d r_{2} d z_{2}\right|^{p} \\
& \quad \leq C\left\{\left\|\sigma^{\prime}\right\|_{\infty}^{2 p}+\left\|\sigma^{\prime \prime}\right\|_{\infty}^{2 p}+\left\|b^{\prime \prime}\right\|_{\infty}^{2 p}\right\} \exp \left\{p C\left(\left\|\sigma^{\prime}\right\|_{\infty}^{2 p}+\left\|b^{\prime}\right\|_{\infty}^{2 p}\right)\right\}
\end{aligned}
$$

Finally, a detailed analysis of the results proved in [Millet and Sanz-Solé (1996)] (see, in particular, Proposition 1.4 and Lemma 2.5) shows that

$$
\left(\lambda^{\bar{h}}\right)^{2}:=\frac{\|\bar{h}\|_{\mathcal{H}}^{2}}{E\left|X_{1}^{0, \bar{h}}(t, x)\right|^{2}} \leq C,
$$

with $C$ depending on $b, \sigma$ and the initial value $X_{0}$. Hence, the remark follows from (3.41), (3.42) and (3.43).

\section{Appendix}

This section is devoted to the proof of some technical ingredients which have been used in the previous sections.

The first Lemma is an exponential inequality for stochastic integrals involving the Green kernel $G_{t-s}(x, y)$. It is an extension of Lemma 2.3 in [Rovira and Tindel (1997)], with a similar proof (see also [Rovira and Sanz-Solé (1996)]). For the sake of completeness we sketch the main arguments.

We denote by $\mathcal{F}_{t}, t \in[0, T]$ the $\sigma$-field generated by $\left\{W_{s, x},(s, x) \in[0, t] \times\right.$ $[0,1]\}$.

Lemma 4.1 Let $\tau=\{\tau(s, y),(s, y) \in[0, T] \times[0,1]\}$ be an $\mathcal{F}_{t}$-adapted, square integrable process. Set

$$
J(t, x)=\int_{0}^{t} \int_{0}^{1} G_{t-s}(x, y) \tau(s, y) W(d s, d y) .
$$

Fix $\beta \in(3, \infty), R>0$ and let $A^{\beta, R}=\left\{\int_{0}^{T} \int_{0}^{1}|\tau(s, y)|^{2 \beta} d s d y \leq R\right\}$. Then, there exist positive constants $K_{1}, K_{2}$ such that

$$
P\left\{\|J\|_{\infty} \geq L, A^{\beta, R}\right\} \leq \exp \left(-\frac{L^{2}}{R^{1 / \beta}} K_{2}\right),
$$

for any $R, L>0$ with $\frac{L}{R^{1 / 2 \beta}} \geq K_{1}$. 
Proof: Fix $0 \leq r \leq t$ in $[0, T], x, z \in[0,1]$. We have

$$
\begin{aligned}
J(t, x)- & J(r, z) \\
= & \int_{0}^{T} \int_{0}^{1}\left(1_{\{s \leq t\}} G_{t-s}(x, y)-1_{\{s \leq r\}} G_{r-s}(z, y)\right) \tau(s, y) W(d s, d y) \\
= & \int_{r}^{t} \int_{0}^{1} G_{t-s}(x, y) \tau(s, y) W(d s, d y) \\
& \quad+\int_{0}^{r} \int_{0}^{1}\left(G_{t-s}(x, y)-G_{r-s}(x, y)\right) \tau(s, y) W(d s, d y) \\
& +\int_{0}^{r} \int_{0}^{1}\left(G_{r-s}(x, y)-G_{r-s}(z, y)\right) \tau(s, y) W(d s, d y) .
\end{aligned}
$$

Let $1 / \alpha+1 / \beta=1$. Notice that $\alpha \in(1,3 / 2)$. Then, by Lemma B.1 in [Bally, Millet and Sanz-Solé (1995)], there exists a constant $C_{0}$ such that

$$
\begin{aligned}
& \max \left(\int_{r}^{t} \int_{0}^{1} G_{t-s}(x, y)^{2 \alpha} d s d y, \int_{0}^{r} \int_{0}^{1}\left(G_{t-s}(x, y)-G_{r-s}(x, y)\right)^{2 \alpha} d s d y\right) \\
& \quad \leq C_{0}|t-r|^{\frac{3-2 \alpha}{2}} \\
& \int_{0}^{r} \int_{0}^{1}\left(G_{r-s}(x, y)-G_{r-s}(z, y)\right)^{2 \alpha} d s d y \leq C_{0}|x-z|^{3-2 \alpha} .
\end{aligned}
$$

Define $\rho(u)=2 C_{0}^{1 /(2 \alpha)} R^{1 /(2 \beta)} u^{1 / 2}, u \geq 0, d((t, x),(r, z))=2 \mid t-$ $\left.r\right|^{(3-2 \alpha) /(2 \alpha)}+|x-z|^{(3-2 \alpha) / \alpha}$,

$$
g(s, y)=\frac{1_{\{s \leq t\}} G_{t-s}(x, y)-1_{\{s \leq r\}} G_{r-s}(z, y)}{\rho(d(t, x),(r, z))} \tau(s, y)
$$

and, for any $0 \leq u \leq T$,

$$
M_{u}=\int_{0}^{u} \int_{0}^{1} g(s, y) W(d s, d y) .
$$

Then, $\left\{M_{u} ; \mathcal{F}_{u}, u \in[0, T]\right\}$ is a continuous martingale with increasing process given by

$$
\langle M\rangle_{u}=\int_{0}^{u} \int_{0}^{1} g(s, y)^{2} d s d y .
$$


By Hölder's inequality, on the set $A^{\beta, R}$

$$
\langle M\rangle_{T} \leq 1
$$

Let $\Psi(x)=\exp \left(x^{2} / 4\right), x \in \mathbb{R}$. Then

$E\left(\Psi\left(\frac{J(t, x)-J(r, z)}{\rho(d((t, x),(r, z)))}\right) 1_{A^{\beta, R}}\right) \leq E_{\tilde{P}}\left\{\exp \left(\frac{1}{4} \sup _{0 \leq u \leq 1}\left|Z_{u}\right|^{2}\right)\right\}=2^{1 / 2}$

where $\left\{Z_{u}, 0 \leq u \leq 1\right\}$ is a Brownian motion defined in some probability space $(\tilde{\Omega}, \tilde{\mathcal{F}}, \tilde{P})$ such that $M_{u}=Z_{\langle M\rangle_{u}}$.

Now, we proceed as in [Sowers (1992)], [Rovira and Tindel (1997)].

Let

$$
B=\iint_{([0, T] \times[0,1])^{2}} \Psi\left(\frac{J(t, x)-J(r, z)}{\rho(d((t, x),(r, z)))}\right) d t d x d r d z .
$$

It follows from (4.2) that

$$
E\left(B 1_{A^{\beta, R}}\right) \leq T^{2} 2^{1 / 2} .
$$

By Garsia-Rodemich-Rumsey Lemma, for the trajectories in $A^{\beta, R}$ we have

$$
\|J\|_{\infty} \leq C_{1} R^{\frac{1}{2 \beta}}\left(\left(\log _{+} B\right)^{1 / 2}+C_{2}\right) .
$$

Moreover, (4.3) yields

$$
E\left(\exp \left(\log _{+} B\right) 1_{A^{\beta, R}}\right) \leq 2+T^{2} 2^{1 / 2}
$$

Finally, from Chebychev's exponential inequality we obtain

$$
\begin{aligned}
& P\left\{\|J\|_{\infty} \geq L, A^{\beta, R}\right\} \\
& \leq \exp \left(-\frac{L^{2}}{R^{1 / \beta}}\left(\left(\frac{1}{C_{1}}-\frac{R^{\frac{1}{2 \beta}}}{L} C_{2}\right)^{2}-\log \left(2+T^{2} 2^{1 / 2}\right) \frac{R^{\frac{1}{\beta}}}{L^{2}}\right)\right)
\end{aligned}
$$

and (4.1) follows whenever $L / R^{1 /(2 \beta)} \geq C_{1} C_{2}$, with $K_{2}=1 /\left(8 C_{1}^{2}\right), K_{1}=$ $\max \left\{2 C_{1} C_{2}, 2^{3 / 2} \log \left(2+T^{2} 2^{1 / 2}\right)^{1 / 2} C_{1}\right\}$. 
Lemma 4.2 Assume (H1) and (H2). Let $h \in K_{y}^{\text {min }}$, then there exists $\lambda^{h}>$ 0 , depending on $h$, such that

$$
\int_{0}^{t} \int_{0}^{1} \dot{h}(s, z) W(d s, d z)=\lambda^{h} X_{1}^{0, h}(t, x)
$$

where $X_{1}^{0, h}(t, x)$ is defined in $(2.2)$.

Proof: We apply the Lagrange multiplier method (see, for instance, Theorem 6.1.1 in [Clarke (1990)]). Therefore there exists $\lambda_{1} \geq 0$ and $\lambda_{2} \geq 0$, not both zero, such that

$$
0 \in\left[\lambda_{1} \partial\|h\|_{\mathcal{H}}^{2}+\lambda_{2} \partial\left\{\psi_{X_{0}}^{h}(t, x)-y\right\}\right](h),
$$

where $\partial$ denotes the generalized gradient.

The mappings $h \rightarrow\|h\|_{\mathcal{H}}^{2}, h \rightarrow \Psi_{X_{0}}^{h}(t, x)$ are continuously Gâteaux differentiable. Thus, the generalized gradients of these mappings reduce to a singleton, the corresponding Gâteaux's derivatives, which are denoted by $\tilde{D}$ in the sequel. One easily checks that $\tilde{D}\|h\|_{\mathcal{H}}^{2}=2 h$ and $\tilde{D} \Psi_{X_{0}}^{h}(t, x) \in \mathcal{H}$ satisfies

$$
\begin{aligned}
\tilde{D}_{\alpha, \beta} \Psi_{X_{0}}^{h}(t, x) & =G_{t-\alpha}(x, \beta) \sigma\left(\Psi_{X_{0}}^{h}(\alpha, \beta)\right) 1_{\{\alpha \leq t\}}+\int_{0}^{t} \int_{0}^{1} G_{t-s}(x, y) \\
& \times \tilde{D}_{\alpha, \beta} \Psi_{X_{0}}^{h}(s, y)\left\{\sigma^{\prime}\left(\Psi_{X_{0}}^{h}(s, y)\right) \dot{h}(s, y)+b^{\prime}\left(\Psi_{X_{0}}^{h}(s, y)\right)\right\} d s d y .
\end{aligned}
$$

Now we prove that $\lambda_{1} \lambda_{2} \neq 0$.

Indeed, suppose that $\lambda_{1}=0$; then $\tilde{D} \Psi_{X_{0}}^{h}(t, x)=0$ which contradicts Lemma 2.5 in [Millet and Sanz-Solé (1996)]. If $\lambda_{2}=0$ then $h \equiv 0$ and $h \in K_{y}^{\min }$. This is contradictory with the assumption $\Psi_{X_{0}}^{0}(t, x) \neq y$. Therefore, by (4.5) there exists $\lambda^{h}>0$ such that

$$
h(\alpha, \beta)=\lambda^{h} \tilde{D}_{\alpha, \beta} \Psi_{X_{0}}^{h}(t, x) .
$$

Using (4.6) one has

$$
\begin{aligned}
\int_{0}^{t} \int_{0}^{1} & \tilde{D}_{s, z} \Psi_{X_{0}}^{h}(t, x) W(d s, d z)=\int_{0}^{t} \int_{0}^{1} G_{t-s}(x, z) \sigma\left(\Psi_{X_{0}}^{h}(s, z)\right) 1_{\{s \leq t\}} \\
& \times W(d s, d z)+\int_{0}^{t} \int_{0}^{1} \int_{0}^{t} \int_{0}^{1} G_{t-\alpha}(x, \beta) \tilde{D}_{s, z} \Psi_{X_{0}}^{h}(\alpha, \beta) \\
& \times\left\{\sigma^{\prime}\left(\Psi_{X_{0}}^{h}(\alpha, \beta)\right) \dot{h}(\alpha, \beta)+b^{\prime}\left(\Psi_{X_{0}}^{h}(\alpha, \beta)\right)\right\} d \alpha d \beta W(d s, d z)
\end{aligned}
$$


Then by Fubini's Theorem and the uniqueness of solution of the involved equations we obtain

$$
X_{1}^{0, h}(t, x)=\int_{0}^{t} \int_{0}^{1} \tilde{D}_{s, z} \Psi_{X_{0}}^{h}(\alpha, \beta) W(d s, d z)
$$

and proves (4.4).

\section{References}

[Bally, Millet and Sanz-Solé (1995)] Baldy, V., Millet, A. and Sanz-Solé, M. (1995). Approximation and Support Theorem in Hölder norm for Parabolic Stochastic Partial Differential Equations. The Annals of Probability Vol 23 1 178-222.

[Bally and Pardoux (1998)] Bally, V. and Pardoux, E. (1998). Malliavin Calculus for white noise driven parabolic SPDEs. Potential Analysis 9 27-64.

[Ben Arous (1988)] Ben Arous, G. (1988). Développement asymptotique du noyau de la chaleur hypoelliptique hors du cut-locus. Ann. Sc. c. Norm. Sup., $4^{e}$ série t $21307-331$.

[Chenal and Millet (1997)] Chenal, F. and Millet, A. (1997). Uniform Large deviations for parabolic SPDEs and applications. Stochastic Processes and their Applications 72 161-186.

[Clarke (1990)] Clarke, F.H. (1990). Optimization and Nonsmooth Analysis. Classics in Applied Mathematics 5. Society for Industrial and Applied Mathematics, Philadelphia.

[Léandre (1988)] LÉandre, R. (1988). Applications Quantitatives et Géometriques du Calcul de Malliavin. In: Lecture Notes in Mathematics 1322 109-133. Springer-Verlag, Berlin.

[Márquez-Carreras and Sanz-Solé (1998a)] Márquez-Carreras, D. and SanzSolÉ, M. (1998). Expansion of the density: A Wiener-Chaos Approach. Bernouilli, to appear.

[Márquez-Carreras and Sanz-Solé (1998b)] Márquez-Carreras, D. and SanzSolÉ, M. (1998). Taylor Expansion of the density in a Stochastic Heat Equation. Collectanea Mathematica, to appear.

[Millet and Sanz-Solé (1996)] Millet, A. and Sanz-Solé, M. (1996). Varadhan Estimates for density of the a Parabolic Stochastic Partial Differential Equation. In: Stochastic Analysis and Applications. A.Truman, I.M. Davies and K.O.Elworthy (Eds), 330-342. Word Scientific Publication. 
[Neveu (1968)] Neveu, J. (1968). Processus Aléatoires Gaussiens. Séminaire de Mathématiques Supérieures (1968). Les Presses de l'Université de Montreal.

[Nualart (1995)] Nualart, D. (1995). Malliavin Calculus and Related Topics. Springer-Verlag.

[Nualart (1998)] Nualart, D. (1998). Analysis on Wiener space and anticipating stochastic calculus. In: Ecole d'été de Probabilités de Saint-Flour XXV (1995). Lecture Notes in Mathematics 1690 123-227.

[Rovira and Sanz-Solé (1996)] Rovira, C. and Sanz-Solé, M. (1996). The Law of the solution to a nonlinear hyperbolic SPDE. Journal of Theoretical Probability Vol 9 863-901.

[Rovira and Tindel (1997)] Rovira, C. and Tindel, S. (1997). Sharp Laplace Asymptotics for SPDEs: The Parabolic and Elliptic cases. Mathematics Preprint Series $\mathbf{2 4 3 .}$

[Sowers (1992)] Sowers, R.B. (1992). Large Deviations for a reaction-diffusion equation with non-gaussian perturbations. The Annals of Probability 20 504-537.

[Takanobu and Watanabe (1993)] TAkanobu, S. and WatanaBE, S. (1993). Asymptotic expansion formulas of the Schilder type for a class of conditional Wiener functional integrations. In: Pitman Research Notes in Math. Series 284, K.O. Elworthy and N. Ikeda (Eds) 194-241.

[Walsh (1986)] WaLsh, J.B. (1986). An Introduction to Stochastic Partial Differential Equations. In: Ecole d'été de Probabilités de Saint-Flour XIV (1984). Lecture Notes in Mathematics 1180 265-439.

[Watanabe (1987)] WatanaBe, S. (1987). Analysis of Wiener functionals (Malliavin Calculus) and its applications to heat kernels. Annals of Probability 15 $1-39$.

Departament d'Economia

Universitat Pompeu Fabra

Ramon Trias Fargas, 25-27

08005 BARCELONA, SPAIN

E-MAIL: kohatsu@upf.es
Facultat de Matemàtiques

Universitat DE BARCELONA

Gran Via, 585

08007 BARCELONA, SPAIN

E-MAIL: marquez@cerber.mat.ub.es

E-MAIL: sanz@cerber.mat.ub.es 